\title{
A Novel Smart Routing Protocol for Remote Health Monitoring in Medical Wireless Networks
}

\author{
T.V.P. Sundararajan ${ }^{1 *}$, PhD, M.G. Sumithra ${ }^{1}$, PhD, R. Maheswar ${ }^{1,2}$, PhD \\ ${ }^{1}$ Department of Electronics and Communication Engineering \\ Bannariamman Institute of Technology, Sathyamangalam 638 401, India \\ ${ }^{2}$ Sri Krishna College of Technology, Coimbatore, 641042
}

Submitted July 2013. Accepted for publication December, 2013.

\begin{abstract}
In a Medical Wireless Network (MWN), sensors constantly monitor patient's physiological condition and movement. Inter-MWN communications are set up between the Patient Server and one or more Centralized Coordinators. However, MWNs require protocols with little energy consumption and the self-organizing attribute perceived in ad-hoc networks. The proposed Smart Routing Protocol (SRP) selects only the nodes with a higher residual energy and lower traffic density for routing. This approach enhances cooperation among the nodes of a Mobile Ad Hoc Network. Consequently, SRP produces better results than the existing protocols, namely Conditional Min-Max Battery Cost Routing, Min-Max Battery Cost Routing and AdHoc Ondemand Distance Vector in terms of network parameters. The performance of the erstwhile schemes for routing protocols is evaluated using the network simulator Qualnet v 4.5.
\end{abstract}

Keywords: selfishness, medical wireless network, routing protocol, energy, routing

\section{INTRODUCTION}

One of the important applications of the complex integration of Medical Wireless Networks (MWN) and advanced wireless technologies is remote health monitoring. In this system, health and motion information is collected in real-time, and sent to nearby storage devices or diagnosis, through which data can be forwarded off-site for further processing. Several design issues must be addressed in order to enable the deployment and adoption of MWNs. From the communications standpoint, it is essential to design appropriate routing protocols to guarantee energy efficiency, higher network capacity, and adequate quality of service (QoS).

Figure 1 shows a general architecture of a MWN-based health monitoring system. Electroencephalography (EEG), Electrocardiography (ECG), Electromyography (EMG), blood pressure sensors and motion sensors, send data to nearby Personal Server (PS) devices. ThroughWLAN/Bluetooth link, these data are streamed remotely to a

*Corresponding author: Dr. T. V. P. Sundararajan, Department of Electronics and Communication Engineering, Bannariamman Institute of Technology, Sathyamangalam 638 401, India. E-mail: suntvp @ bitsathy.ac.in. Other authors:mgsumithra@rediffmail.com,maheshh3@rediffmail.com. 


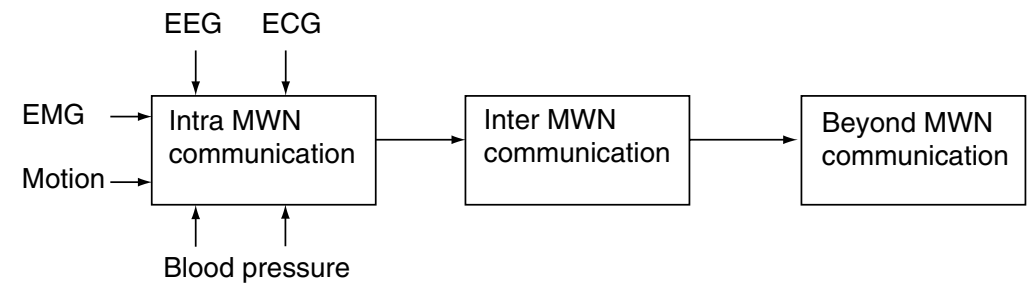

Figure 1. Architecture of health monitoring communication System.

medical doctor's site for real time opinion, to a health check database for record maintenance, or to acorresponding equipment that issues acrisis alert. Intra-MWNradio communications, taking place in about 2 meters around the patient's body, includes: (1) communications among body sensors, and (2) communications between body sensors and the portable PS. On the other hand, inter-MWN communications are defined as the communications between the PS and one or more Centralized Coordinators (CCs). The CCs can be organized as part of the infrastructure, or be tactically placed in a dynamic environment for handling emergency situations [1]. The prototype of inter-MWN communications can be divided into two categories, infrastructure-based architecture as shown in Figure 2 and ad hoc-based architecture as shown in Figure 3. While the infrastructure-based architecture offers larger bandwidth with centralized management and flexibility, the adhoc-based architecture facilitates rapid deployment in a dynamic environment, such as medical crisis care response, or catastrophe site [2].

In the ad hoc based architecture, multiple CCs are set up to facilitate the body sensors broadcast information with in hospital centers. Therefore, the service coverage is larger than in the infrastructure-based architecture, helping users to move around in

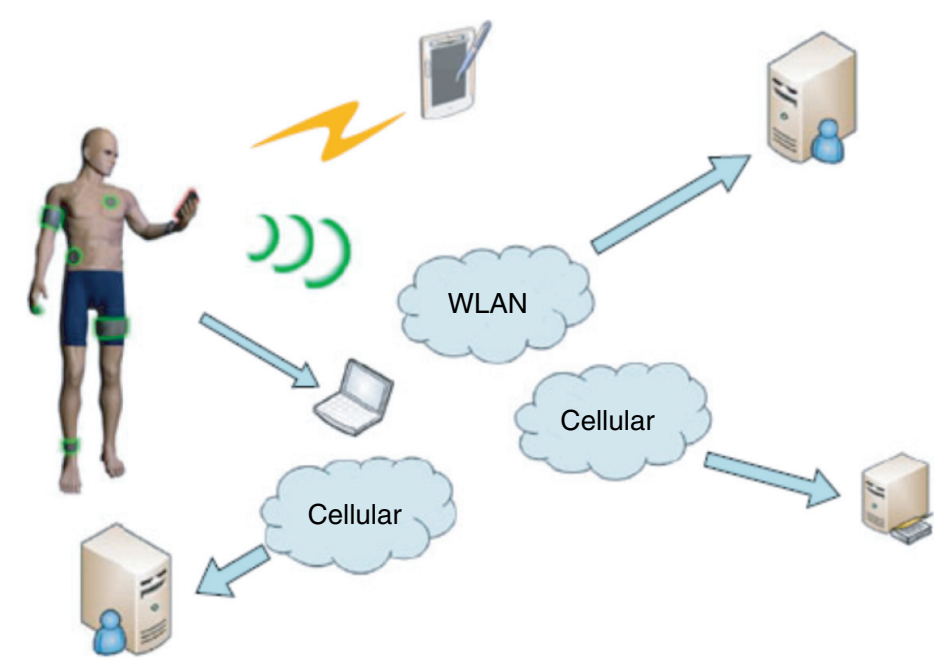

Figure 2. Inter-MWN communication architecture: infrastructurebased mode. 


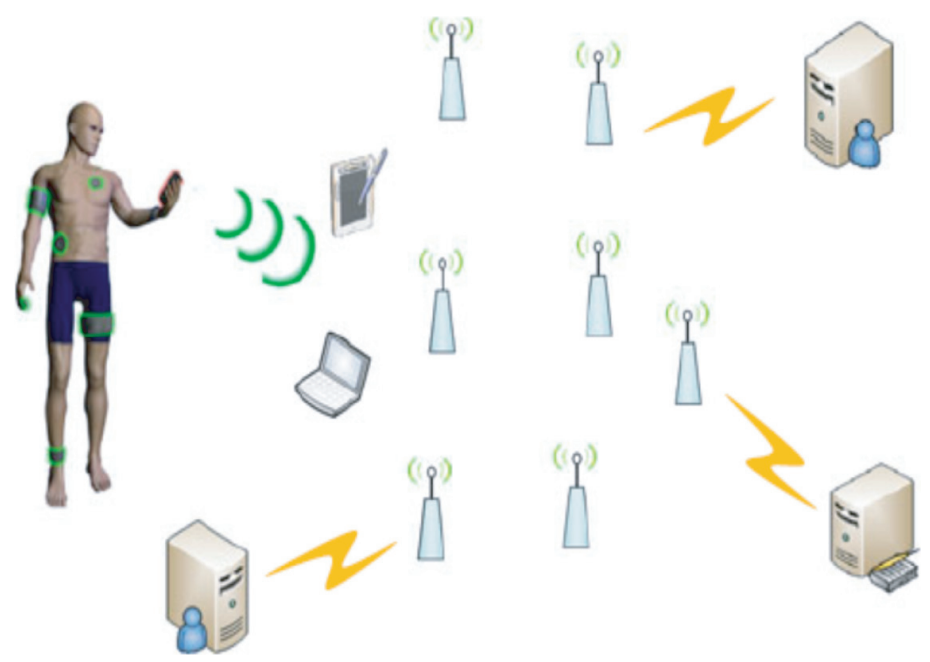

Figure 3. Inter-MWN communication architecture: ad hoc based mode.

a building, playground, or in an emergency rescue spot. While the coverage of an MWN is restricted to about two meters, this wayof interconnection widens the system to about one-hundred meters, which suits both in short-term and long-term setups. Since there is only one radio, all communications share the same bandwidth; therefore, lack of cooperation can easily occur, given that the number of routers and sensor/actuator nodes is larger in certain areas.

\subsection{Objectives}

In SRP-based ad hoc network, various mobile devices with different objectives share their resources in order to ensure global connectivity. However, some resources are expended quickly as the devices partake in the network functions. For example, battery power is rated to be the most significant resource in a mobile environment. A peculiar mobile device may strive to take advantage from other devices, and reject to share its own resources. Such nodes are called selfish or misbehaving nodes and their behavior is termed selfishness or misbehavior [3]. One of the foremost causes of energy expenditure in the mobile nodes of mobile ad hoc network (MANET) is wireless transmission [4]. A selfish node ( $\mathrm{SN}$ ) may snub andtransmit data packets to other nodes to conserve its own energy. SNs exploit the network but do not assist others, with a view to hoard battery life for their own communications, although SNs do not aim to directly ruin other nodes.

Countermeasures against device selfishness and misbehavior are essential in mobile ad hoc networks. Selfishness lack of node support cannot be solved by traditional security techniques [5]. In this article, Smart Routing Protocol (SRP) is propounded. The main objective of SRP is to ensure that a node with a low residual energy and high traffic density is not selected for routing. The cost metric used in the SRP routing protocol take into account not only the residual battery capacity of a node but also the 
drain rate and power consumption in transmitting a packet to increase the battery life of nodes [6]. This approach prevents a node from becoming selfish in a short time and enhances cooperation among the nodes of an MANET. In SRP, each node checks the remaining battery capacity and determines the activity of route construction process. The effect of power consumption and traffic density of a node is also captured by the drain rate in this SRP protocol.

\section{RELATED WORKS}

SRP ad hoc networks are multi-hop networks. In ad hoc networks, energy conservation is a significant factor not only to increase the life of the nodes but also to avert the node from becoming selfish in the network. Augmenting cooperation among nodes is thus a crucial issue in such networks. In principle, energy conservation can be attempted at different layers of networking protocols. The proposed SRP protocol emphasizes on the network layer.

Standard routing algorithms such as Adhoc On-demand Distance Vector (AODV), Dynamic Source Routing (DSR) disregard the residual battery power of the nodes [7]. In general, the links between two nodes are set up through the shortest path routes. Such algorithms may cause quick depletion of the battery power in the nodes, that are common to the heavily used routes in the network. Sooner or later, the node with depleted battery will be reluctant to participate or withdraw itself fromthe existing routing andleadingto DoS selfish attack.

Wireless sensor networks (WSNs) perform data collection with the objective of obtaining the whole dataset at the sink. Many data aggregation schemes have been investigated [8]; they either compromise the fidelity of the recovered data or require complicated in-network compressions. A novel data aggregation scheme exploits compressed sensing (CS) to achieve both recovery fidelity and energy efficiency in WSNs with arbitrary topology [8]. A multi-constrained multicast routing protocol was suggested based on genetic algorithm that determines near-optimal multicast routes on demand. This proposed protocoltried to optimize multiple QoS parameters simultaneously [9]. Further research is needed to reduce works on the intermediate nodes and improve the design principle of routing protocols.

The lifetime of a network is characterized as the duration from the time whenthe network commences functioning until the first node runs out of battery charge. As the preference of this network lifetime obviously directs to a max-min type (bottleneck) optimization problem, it is required to maximize the first node failure (minimum) time. The network layer solutions for battery management aim mainly at increasing the lifetime of the network. The major solutions provided in the literature focus primarily on developing routing protocols that use routing metrics such as low energy cost and remaining battery charge [10]. Cross-layer optimization techniques were developed that extend DSR to improve its routing energy efficiency by minimizing the frequency of recomputed routes. However, this approach will only check the last received signal strength, the DSR will not invoke the route maintenance phase causing an infinite loop of maintenance messages [11].

One of the main issues concerning ad hoc wireless networks is the relay traffic. Each node deals with two kinds of traffic, relay traffic and its own traffic. A trade-off is 
reached between the blocking probability of the relay traffic and the battery efficiency. The intermediate node may not want to transmit the entire neighbour's traffic. Whenever a traffic session is generated at the source node, a route with minimum energy cost is selected. An intermediate node can allow the session traffic by sending an acknowledgement to the source node. If the latter is chosen, on receiving a negative acknowledgement, the source repeats the process for the next best route on the basis of energy cost. If all the routes are blocked, the session is taken to be blocked. Each node tries to behave selfishly when there is relay traffic. The relay node rejects relay traffic based on total quantity of data the source intends to send to the destination and the strategy used [12]. The number of packets dropped by the relay nodes decreases as the number of selfish user decreases.

Establishing trust among distributed network entities has been recognized as a powerful tool to improve the security and performance of distributed networks such as MANETs and sensor networks. In general, the resource constraints of medical sensor nodes make the use of many solutions including asymmetric cryptosystems unrealistic or impossible in the majority of circumstances. Further, these approaches cannot pin point exactly from where the false information is introduced into the network and who is responsible for it $[13,14]$.

Recently, multi-hop secondary networks have gained attention as a promising design to leverage the full potential of cognitive radio networks (CRNs). One of the main features of routing protocols in multi-hop networks is the routing metric used to select the best route for forwarding packets. Various challenges were listed in designing a routing metric for CRNs, both inherited from traditional wireless networks and those unique to CRNs [15]. The escalating energy costs and environmental concerns have already created an urgent need for more energy-efficient "green" wireless communications. Remarkable techniques were discussed toward green mobile networks to date, mainly targeting mobile cellular networks in [16]. Moreover, the issues of node failure and power efficiency should be taken into consideration and the mathematical model has to be designed for fault-tolerant topology control problem in wireless sensor networks [17].

Minimizing the overall transmission power and distributing the power consumption evenly among the nodes are contradictory to each other. The routing algorithms such as Conditional Min-Max Battery Cost Routing (CMMBCR), Min-Max Battery Cost Routing (MMBCR) attempted to find a balance between two factors by using new node metrics for the route discovery process. A power optimal scheduling and routing protocol was promoted to reduce the total average power in the network, subject to limitations such as peak transmission power of the devices and attainable data rate per link [18]. Forwarder nodes were set up in order to extend the lifetime only in sensor networks. It was assumed that the battery of a node can be recovered when it was allowed to rest. However, this may not be the real scenario in sensor networks [19].

Energy Efficient Optimized Link State Routing (EOLSR) was the energy efficient extension of Optimized LinkState Routing (OLSR). Like OLSR, EOLSRconsists of two main functionalities: neighborhood discovery and topology dissemination [20]. EOLSR was based on an energy model selecting energy-aware multipoint relays (EMPRs), computes energy-efficient routes and optimizes broadcasts. As EOLSR was a proactive based protocol, its time complexity and cost complexity will not be 
guaranteed compared to our proposed SRP. As the threshold energy level was set to only $10 \%$ of initial energy of 50 joules, EOLSR has to repeat the route selection process frequently. However, SRP uses the dynamic threshold value; it reduces the probability of repeated route selection process.

A novel routing protocol algorithm was proposed for wireless sensor network in [21]. Due to limitations of power, computation capability and storage resources of wireless sensor networks are vulnerable to many attacks. Despite the simulation results, the proposed routing protocol algorithm in [21] can adopt suitable routing technology only for the nodes according to the distance of nodes to the base station, density of node distribution and residual energy of nodes. This routing technology did not consider attacks such as selfish attack as in case of SRP.

\section{PROPOSED SMART ROUTING PROTOCOL}

In this paper, an SRP is proposed that can increase the survivability of nodes as well as network by avoiding nodes with low residual battery power (i.e., potential selfish nodes). To achieve this aim, a cost metric that optimizes energy utilization in the network has been developed. The concept of drain rate has also been incorporated in the cost metric to capture the effect of current load on the node. A realistic battery model is assumed to characterize the recovery effect observed in the batteries.

\subsection{Battery Model}

In traditional routing protocols, the battery is assumed to have linear charge and discharge characteristics. On the other hand, real battery drains more rapidly when higher loads are applied. The life span of a battery and its delivered power mainly depend on the current discharge profile [22]. If the discharge current magnitude in a battery is higher than its rated current capacity, it supplies smaller amount of energy. This effect is termed as "rate capacity effect" which results from the fact that at a higher discharge rate, electrochemical reductions occur only at the outer surface of the cathode. This implies that a battery can deliver more energy if the rate of current drawn is lower. The lifetime of a battery can also be increased by the alternating incidences of idle and discharge phases. During the idle periods, the battery partially recovers its lost capacity. This consequence is known as "recovery effect" [22]. It is a phenomenon that the charge recovery rateis higher initially but tapers off with time. A simple instinctive function as in equation (1) is used here to empirically capture the recovery effect in the battery model as suggested by [23]:

$$
r(t)=c_{1} e^{-c_{2} t}
$$

where $c_{1}$ and $c_{2}$ are constants, $t$ is time, and $r(t)$ is the recharge rate of the battery. It is assumed that the battery recharges at a rate of $d(t, v(t))$ when in use, and recharges at a rate of $r(t, v(t))$ whenat rest, where $v(t)$ is the voltage of the battery at time t. In general, different batteries have different functions of $r(t)$ and $d(t)$. The values of constants $c_{1}$ and $c_{2}$ depend upon the type of battery technology used. However, it is assumed that the 
discharge rate remains constant, i.e. $d(t, v(t))=c_{3}$, for the simulation, as the discharge relies on the size of the packet being transmitted and not on current voltage and past usage.

\subsection{Basic Assumptions}

SRP is a reactive routing protocol, where routing verdicts are made at each hop. The routes acquired by a data packet are based on the cost of routing that packet. The proposed cost metric ensures that a node with high residual battery energy and low traffic density is highly preferred during routing progression. On the contrary, a node with low residual battery energy and higher traffic density is not selected for routing. The following basic assumptions made in SRP are based on the characteristics of the MANET nodes and the battery:

- $\quad$ Each device can estimate its own remaining battery power.

- $\quad$ Each node can assess how much power is consumed in transmitting a packet.

- $\quad$ Each node knows its current battery drain rate.

- A battery recovers some of its lostcapacity during rest between discharges, and its life is elongated because of the charge recovery effect. Note that this assumption is applicable only to MANET where nodes are similar to laptops. They use batteries that have huge capacity and are rechargeable, such as Lithium Polymer batteries of very smallform factor, a promising technology. Rechargeable batteries could considerably expand the lifetime of a network.

- In sensor networks, one of the controlling features is its battery life. A very attractive attribute of sensor networks would be its rechargeability to extend functioning over a longer period of time. On the other hand, the nodes craving recharge may not be accessible. By adopting a battery recharge mechanism such as variable power infrared (IR) laser, recharging canbe achieved acrossdistances up to several hundred meters in ahard access environment [24].

- Each node has identical initial energy level and the battery capacity of each host is the same.

\subsection{Route Selection Cost Metric}

Every node, except the destination node, estimates its cost, $C_{i}$, by the equation below:

$$
C i(t)=P\left(\frac{F i}{R i(t)}\right) D R i
$$

where $P$ is the power consumed in transmitting a packet, $F_{i}$ is the full battery capacity of node $i, D R_{i}$ is the drain rate of node $i$ calculated by exponential weighted moving average method[25]. $R_{i}(t)$ is the residual battery capacity of the node $i$ at time $t$, and is given by:

$$
R i(t)=R i\left(t-t_{i}\right)+\text { charge recovered in time } t_{i}
$$


where $t_{i}$ is the idle time. This cost metric assists in discovering the links with the least cost nodes, so that data packets can shun the nodes with quick depleting batteries. The cost given by this metric is directly proportional to the power consumed in routing a packet in a node, and inversely proportional to the normalized residual battery capacities of these nodes. Therefore, nodes with higher traffic densities are spared during routing. These are the three basic operations in SRP: route discovery, data routing, and route maintenance, which are described in the following sections.

\subsection{Route Discovery}

SRP is a reactive routing protocol that can construct the route when data transmission is required. In this protocol, a source node broadcasts the Route Request (RREQ) packet to the entire network, and all the nodes rebroadcast the received RREQ packet immediately. When a source device transmits data to a destination device whose route is unknown, it relays an RREQ along with its coordinates to its neighbors. It also assigns sequence numbers to this RREQ packet so as to differentiate between accomplished and new sessions. The intermediate nodes that have residual energy greater than the threshold $\left(\mathrm{R}_{\mathrm{th}}\right)$ retransmit this packet. This threshold $\left(\mathrm{R}_{\mathrm{th}}\right)$ is decreased with the progress of time to ensure that all nodes have their residual battery chargeat the same level. The destination, on receiving an RREQ packet, adds that to its route reply list and broadcasts a Route Reply (RREP) packet. The intermediate nodes act as cooperative nodes and forward these RREP packets towards the source only when their residual energy is greater than the threshold $\left(\mathrm{R}_{\mathrm{th}}\right)$. The following distance condition is satisfied; otherwise, the nodes become selfish nodes and the RREP packets are dropped:

$$
\begin{aligned}
& d\left(N_{i}, N_{s}\right) \leq d\left(N_{j}, N_{s}\right) \\
& d\left(N_{i}, N_{d}\right) \geq d\left(N_{j}, N_{d}\right)
\end{aligned}
$$

whered $\left(\mathrm{N}_{\mathrm{i}}, \mathrm{N}_{\mathrm{j}}\right)$ is the distance between the pairs of nodes $\mathrm{N}_{\mathrm{i}}$ and $\mathrm{N}_{\mathrm{j}}$; and $\mathrm{N}_{\mathrm{i}}, \mathrm{N}_{\mathrm{j}}, \mathrm{N}_{\mathrm{s}}$ and $\mathrm{N}_{\mathrm{d}}$ are the current, previous hop, source, and destination nodes, respectively.

In this SRP protocol, each node is involved in route construction in accordance with the remaining battery capacity. Each node adjusts the retransmit timing of the RREQ packet since the RREQ packet that arrives first is used to construct the route. The retransmit timing is determined by the remaining battery capacity. Therefore, when the node accepts the RREQ packet from a new node, it initiates the timer for the retransmit of the received RREQ packet by verifying the residual battery capacity. The timer period is fixed to a large value when the battery capacity is small. Conversely, it is fixed to a small value when the battery capacity is large. The destination, on receiving an RREQ packet, adds that to its route reply list and broadcasts the RREP so that all the intermediate nodes can use this information to check the distance condition. Intermediate nodes forward these RREP packets towards the source only when their residual energy is greater than the threshold value; otherwise the RREP packets are dropped. 
Redundant retransmits often cause the node to waste battery power. In the SRP protocol, until the timer timeout, each node interrupts the retransmit process of the received RREQ packet when the same RREQ packet is received. SRP protocol can avoid the flooding of the RREQ packets and reduce the power consumed to retransmit the redundant RREQ packets.

\subsection{Data Routing}

Once a route to the destination is identified, the nodes (including the source) relay the data packets as per their routing information, using the least cost route. When a source node does not need a session any longer, it signals for the cessation of the session by transmitting a "Finish Session" packet. On getting this Finish Session packet, the intermediate nodes with a matching entry in their routing tables delete that entry from their tables. Ultimately, when the destination node receives this packet, it removes this session from its route reply list.

\subsection{Example Scenario of Routing Algorithm}

Figure 4 shows an example of scenario of smart routing on a typical ad hoc network topology of medical wireless nodes (Personal Server of each patient) and the operations in the SRP protocol. In the example, the network comprises seven Personal Servers (PS) and one destination node (Control Station of Hospital). The value under each PS in Figure 4 indicates the remaining battery capacity. In the proposed protocol, a node that has a large value of the remaining battery capacity constructs the route actively.

Figure 5 further shows the steps of a smart routing algorithm for the example of Figure 4, including the following:

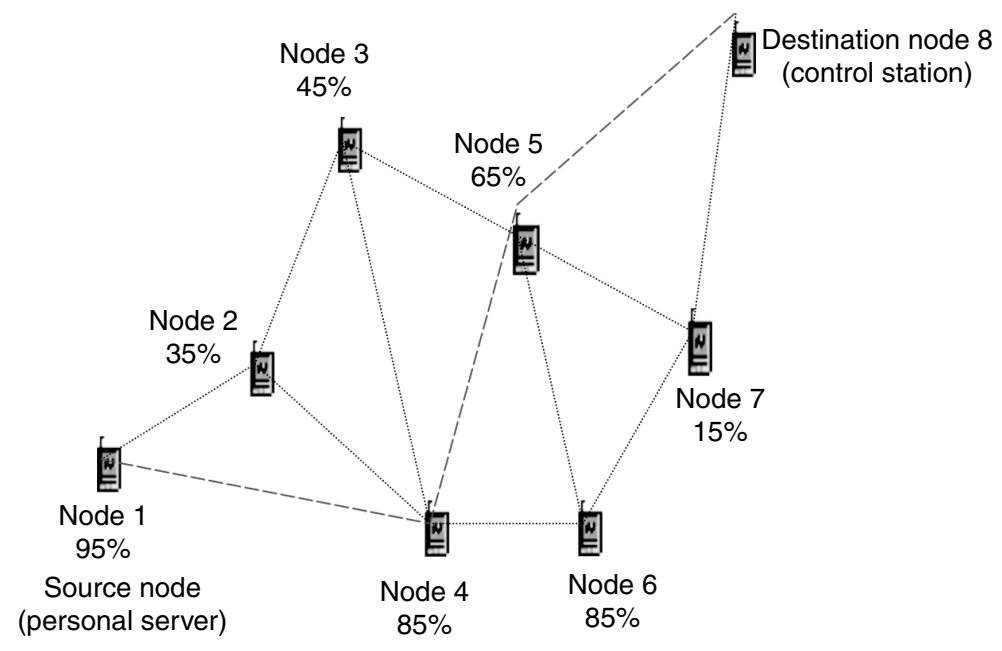

Figure 4. An example of route construction of the SRP protocol. 


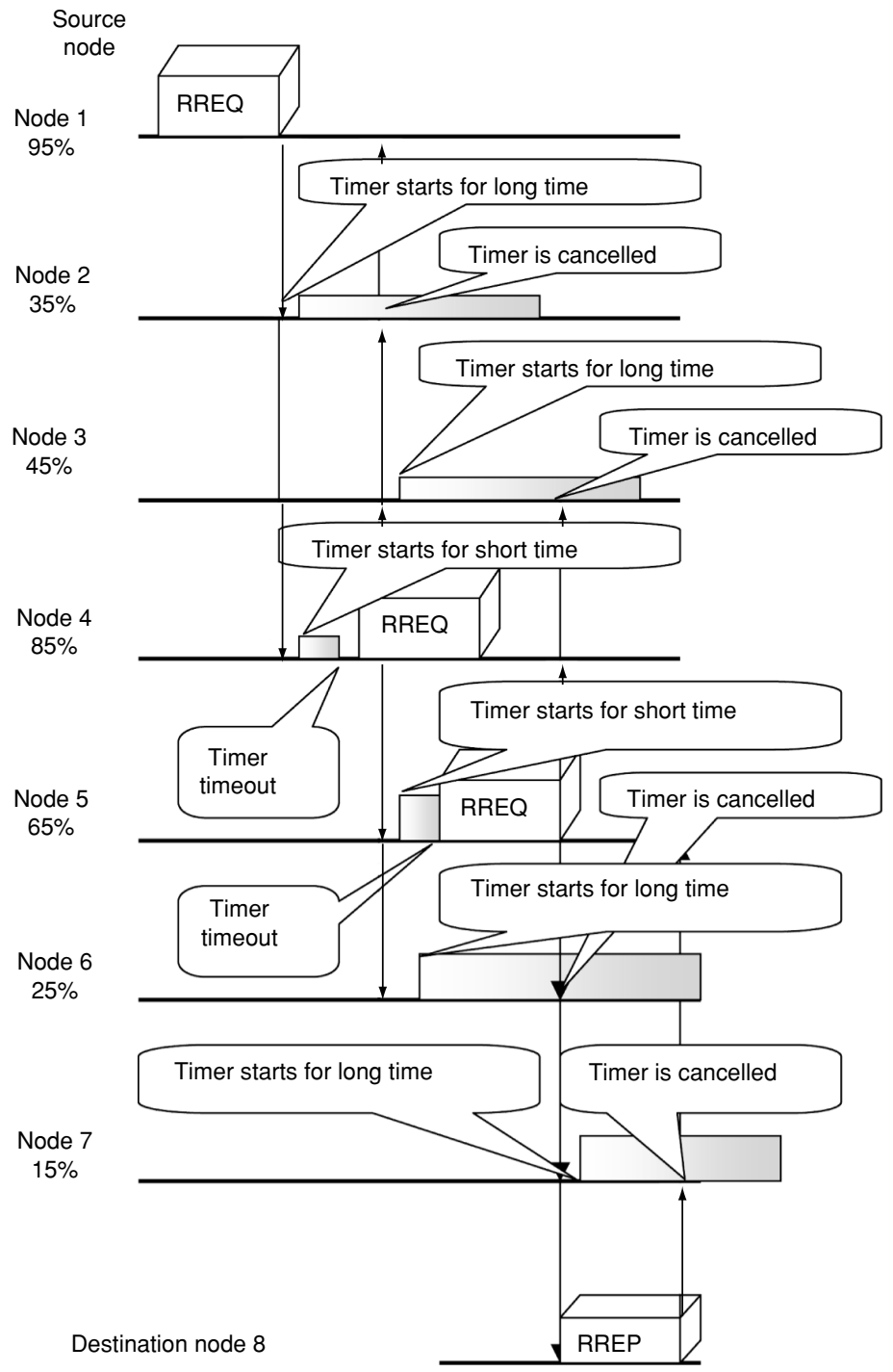

Figure 5. An example of operations in the SRP protocol.

1) Whenever a PS of a patient senses any physiological parameter crosses the threshold level, it immediately sends an alarm signal and the patient's medical data to hospital authority server (Control station). Suppose the patient is away from the authority server, the concerned PS will search reliable routes for communication to broadcast the information to the hospital authority server. In this example, Node 1 is a source node that initiates a route construction process for its emergency purpose. 
2) Node 1 broadcasts an RREQ packet to the network to find a route to the destination node 8 .

3) Nodes 2 and 4 that receive the RREQ packet from node 1 set a timer with a duration that depends on the remaining battery capacity.

4) Node 4 rebroadcasts the RREQ packet in advance, since the remaining battery capacity of node 4 is greater than that of node 2 .

5) Node 2 receives the RREQ packet from node 4 and detects that node 4 retransmits the RREQ packet in advance. Therefore, it stops the timer and interrupts the rebroadcast of the RREQ packet.

6) Nodes 3, 5, and 6 that receive the RREQ packet from node 4 set a timer with the same duration as that of nodes 2 and 4 .

7) Node 5 rebroadcasts the RREQ packet in advance since the remaining battery capacity of node 5 is greater than that of nodes 3 and 6 .

8) Nodes 3 and 6 receive the RREQ packet from node 5 and detect that node 5 has retransmitted the RREQ packet. Therefore, they stop the timer and interrupt the rebroadcast of the RREQ packet.

9) The destination node (Control station) 8 receives the RREQ packet from node 5 and replies with a route reply (RREP) packet to source node. Finally, the route via intermediate nodes 4 and 5 is constructed.

\subsection{Route Maintenance}

Periodic route maintenance is required for the following reasons:

- Some links between the nodes in the routing table may cease to exist due to node movements.

- The battery of a few nodes in the routing map may drain too quickly [25].

- Route maintenance and dissemination of the cost information of the nodes are done periodically by flooding RREP packets from each of the destinations. The periodicity of the retransmission of these RREP packets is critical for the efficient operationof this protocol. It has been resolved through simulation experiments using Qualnet network simulator. These new RREP packets establish the new routes that might have been formed since the last RREP packet was sent, and also help in removing the links that were broken during this period. Each intermediate node in the path also monitors the decrease in its remaining energy level, dropping below a certain threshold level $\left(\mathrm{R}_{\mathrm{th}}\right)$. It then transmits a Route Error (RERR) packet to its nearby nodes, which update their routing tables accordingly. However, this verdict of route repairs is dependent on the lingering battery capacity of the current node and hence, it is a local decision.

The main advantages of SRP over the reported routing protocols in the literature are the following:

- $\quad$ SRP does not necessitate comprehensive information about the energy of all the nodes to be transmitted in the network. All the nodes on the route are vigilant of their traffic densities and energy grades more consistently. Thus, the nodes with an excessive residual energy already on some routes are not preferred in route selection.

- $\quad$ SRP traces multiple routes during route search. It helps in identifying alternative connectivity between the source and the destination. 
- $\quad$ SRP uses a simple intuitive empirical function to capture the charge recovery effect observed in the batteries of mobile nodes, and the values of various constants for different types of batteries are obtained by simple experiments.

- The dynamic variation of the threshold $\left(\mathrm{R}_{\mathrm{th}}\right)$ function ensures that all the nodes consume about the equal amount of energy, so that they maintain approximately the same energy levels. The node that consumes its energy too rapidly will be protected from routing, while others will be encouraged. In this way, energy consumption will be balanced in the whole network.

\section{RESULTS AND DISCUSSIONS}

The performance of the SRP protocol is evaluated using Qualnet v4.5 network simulator. For this purpose, the network lifetime, packet delivery ratio, average end-toend delay, average remaining battery capacity, and active cooperative nodes are used as metrics to compare the performance of SRP with CMMBCR, MMBCR and AODV routing protocols introduced in section 2 . Table 1 lists the simulation factors and environment tested.

\subsection{Simulation Environment}

The Qualnet v4.5 network simulator was employed for the current simulation studies. All the simulations were performed at the network layer by means of a routing agent plugged on top of the Medium Access Layer (MAC) layer agent. The layer above the routing layer calls the routing agent when it has to send a packet to a given node in the network. It is the task of the routing unit to find the next hop for the packet before it forwards the packet down to the MAC layer unit.

Table 1. Simulation parameters

$\begin{array}{ll}\text { Simulator } & \text { Qualnet v } 4.5 \\ \text { Number of nodes } & 60 \text { nodes } \\ \text { Area } & 800 \mathrm{~m} \times 800 \mathrm{~m} \\ \text { Node placement } & \text { Random } \\ \text { Node mobility } & \text { Static (pause time of } 300 \mathrm{sec} \text { ), low (pause } \\ & \text { time of } 100 \mathrm{sec} \text { ), medium (pause time of } 60 \\ & \text { sec) and high (pause time of } 20 \mathrm{sec} \text { ). } \\ \text { Traffic pattern and load } & \text { CBR traffic at rates of } 8 \mathrm{kbps} \text { and } 56 \mathrm{kbps} \\ \text { Data packet size } & 512 \text { bytes } \\ \text { MAC } & \text { IEEE } 802.11 \\ \text { Bandwidth } & 2 \mathrm{Mbps} \\ \text { Transmission range } & 250 \mathrm{~m} \\ \text { Battery capacity } & 1000 \text { Joules } \\ \text { Propagation path loss model } & \text { Free space } \\ \text { Routing protocols } & \text { SRP, AODV, CMMBCR, MMBCR }\end{array}$


The MAC layer protocol used for this study was the IEEE 802.11 protocol with User Datagram Protocol (UDP) as the underlying transport agent. All data packets was generated using an application that generated packets at a Constant Bit Rate (CBR). The power required to transmit $\left(\mathrm{P}_{\mathrm{txt}}\right)$ and receive $\left(\mathrm{P}_{\mathrm{rec}}\right)$ a message is given by eqn. 6 and eqn.7:

$$
\begin{gathered}
\mathrm{P}_{\mathrm{txt}}=\mathrm{p}_{\mathrm{tx}} \cdot \mathrm{t}_{\mathrm{tx}} \\
\mathrm{P}_{\mathrm{rec}}=\mathrm{p}_{\mathrm{rx}} \cdot \mathrm{t}_{\mathrm{rx}}
\end{gathered}
$$

where the probability of transmitting $\left(\mathrm{p}_{\mathrm{tx}}\right)$ and the probability of receiving $\left(\mathrm{p}_{\mathrm{rx}}\right)$ a packet are takenas 0.8 and 0.4 , respectively; the time required to transmit and the time required to receive a packet are $t_{\mathrm{tx}}$ and $\mathrm{t}_{\mathrm{rx}}$, respectively. It may be noted that the more conflict, the longerthe time expended in transmitting/receiving the packets, and hence more power consumed in transmitting the packets.

The source and destination pair were randomly chosen among all the nodes in the network so that each node had a chance to connect to every other node. The simulation time was set to 5000 seconds so that the system can reach steady states. The threshold value $(\gamma)$ for CMMBCR was set at $75 \%$ of the initial energy. Extensive simulation experiments were carried out to comparethe performance of the current SRP with CMMBCR, MMBCR and AODV routing protocols. A total of $2 \times 4 \times 2=16$ scenarios $\{($ small, large networks $) \times(4$ levels of mobility $) \times($ low, high data rates $)\}$ were implemented to test the efficiency of these protocols. For each data point, 30 simulations were run to obtain the average value. The links between the nodes were set and broken at different points of time for different durations. The connection was set up randomly for all the scenarios. The mobility model was based on the random way point model. In this model, a node travels at a certain speed in a given direction for a certain period of time. Subsequently, it pauses at the destination for a given period of time, and again resumes its journey in another possible direction at a different speed. In this simulation study of mobile networks, the speed chosen range between 1 and $10 \mathrm{~m} / \mathrm{s}$. The pause time varied according to the degree of mobility required.

\subsection{Network Life Time}

Network Life Time (NTL) indicates the time of death of the first node. Figure 6 indicates that the NTL of SRP is $12 \%, 12.7 \%$ and $84 \%$ longer compared to that of CMMBCR, MMBCR and AODV, respectively, at a high mobility for 8 kbps traffic rate. The NTL of SRP is $6.9 \%, 8 \%$ and $85 \%$ longer than the NTL of CMMBCR, MMBCR and AODV, respectively, at a medium mobility for $8 \mathrm{kbps}$ traffic rate. The NTL of SRP is $5.3 \%, 6.6 \%$ and $76.4 \%$ longer than the NTL of CMMBCR, MMBCR and AODV, respectively, at a low mobility for $8 \mathrm{kbps}$ traffic rate. The NTL of SRP is $7.2 \%, 7.1 \%$ and $74.8 \%$ longer compared to the NTL of CMMBCR, MMBCR and AODV, respectively, at no mobility (static) for $8 \mathrm{kbps}$ traffic rate. 


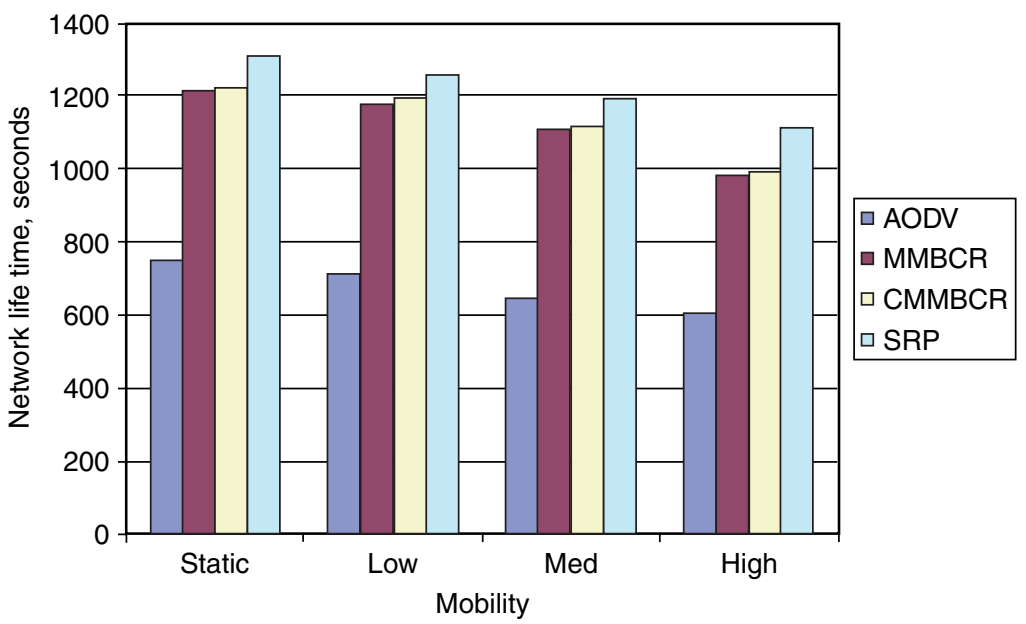

Figure 6. Network life time at $8 \mathrm{kbps}$ traffic rate.

Figure 7 shows that the NTL of SRP is $16.5 \%, 17.8 \%$ and $72 \%$ longer than the NTL of CMMBCR, MMBCR and AODV, respectively, at a high mobility for $56 \mathrm{kbps}$ traffic rate. The NTL of SRP is $18 \%, 20 \%$ and $66 \%$ longer compared to the NTL of CMMBCR, MMBCR and AODV, respectively, at a medium mobility for $56 \mathrm{kbps}$ traffic rate. It is also observed that the NTL of SRP is $20.2 \%, 21 \%$ and $53.9 \%$ longer than the NTL of CMMBCR, MMBCR and AODV, respectively, at a low mobility for 56 kbps traffic rate. The NTL of SRP is also $15.4 \%, 15.7 \%$ and $43.8 \%$ longercompared to the NTL of CMMBCR, MMBCR and AODV, respectively, at a static scenario for $56 \mathrm{kbps}$ traffic rate.

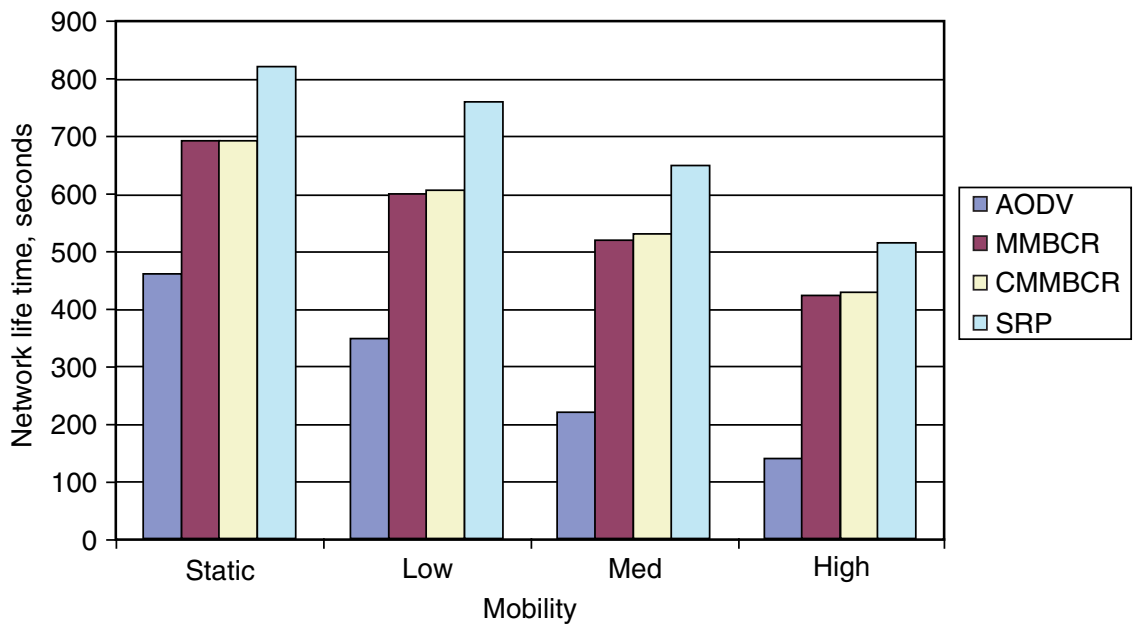

Figure 7. Network life time at $56 \mathrm{kbps}$ traffic rate. 
Figures 6 and 7 suggest that the SRP protocol increases the network lifetime beyond the NTLs of AODV, MMBCR and CMMBCR at all levels of mobility. This can be attributed to the fact that nodes with low residual battery power and higher drain rate are protected in SRP from further participation in routing activities, and due to the battery charge recovery effect, those batteries regain a portionof the lost capacities.

\subsection{Packet Delivery Ratio}

Figure 8 demonstrates the effect of mobility on the Packet Delivery Ratio (PDR) of SRP, AODV, MMBCR and CMMBCR protocols. PDR of the SRP is $6.8 \%, 9.3 \%$ and $16.2 \%$ higher compared to the PDRs of CMMBCR, MMBCR and AODV, respectively, at a high mobility for $8 \mathrm{kbps}$ traffic rate, as shown in Figure 8. The PDR of SRP is 5.5\%, $8.5 \%$ and $16.9 \%$ higher compared to the PDRs of CMMBCR, MMBCR and AODV, respectively, at a medium mobility for $8 \mathrm{kbps}$ traffic rate. It is also shown that the PDR of SRP is $2 \%, 4.4 \%$ and $11.2 \%$ higher than the PDRs of CMMBCR, MMBCR and AODV, respectively, at a low mobility for $8 \mathrm{kbps}$ traffic rate. The PDR of SRP is $1.8 \%$, $4.4 \%$ and $9.7 \%$ higher compared to the PDRs of CMMBCR, MMBCR and AODV, respectively, at no mobility for $8 \mathrm{kbps}$ traffic rate.

Figure 9 shows the effect of mobility on the Packet Delivery Ratio (PDR) of SRP, AODV and MMBCR protocols. The PDR of SRP is 2.2\%, 7.8\% and $11.6 \%$ higher than the PDRs of CMMBCR, MMBCR and AODV, respectively, at a high mobility for 56 kbps traffic rate. The PDR of SRP is $4.7 \%, 12.2 \%$ and $16.4 \%$ higher compared to the PDR of CMMBCR, MMBCR and AODV, respectively, at a medium mobility for 56 kbps traffic rate. Figure 9 also demonstratesthat the PDR of SRP is $1.9 \%, 4.2 \%$ and $5.1 \%$ higher than the PDR of CMMBCR, MMBCR and AODV, respectively, at a low mobility for $56 \mathrm{kbps}$ traffic rate. The PDR of SRP is $0.4 \%, 2.7 \%$ and $5.8 \%$

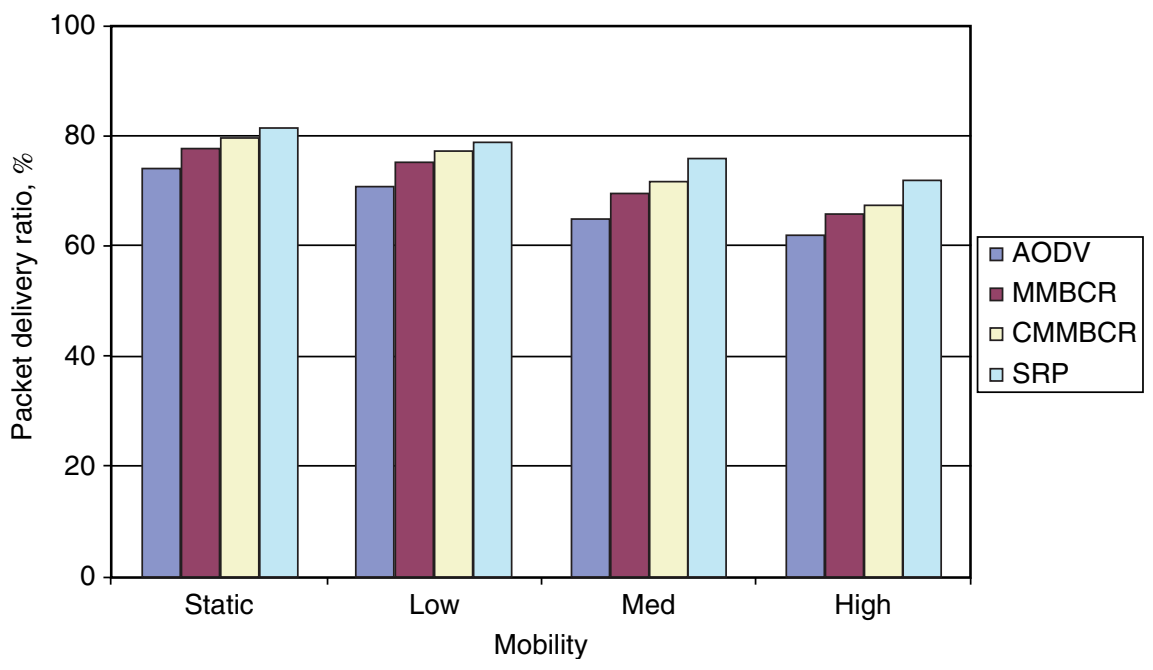

Figure 8. Packet delivery ratio at 8 kbps traffic rate. 


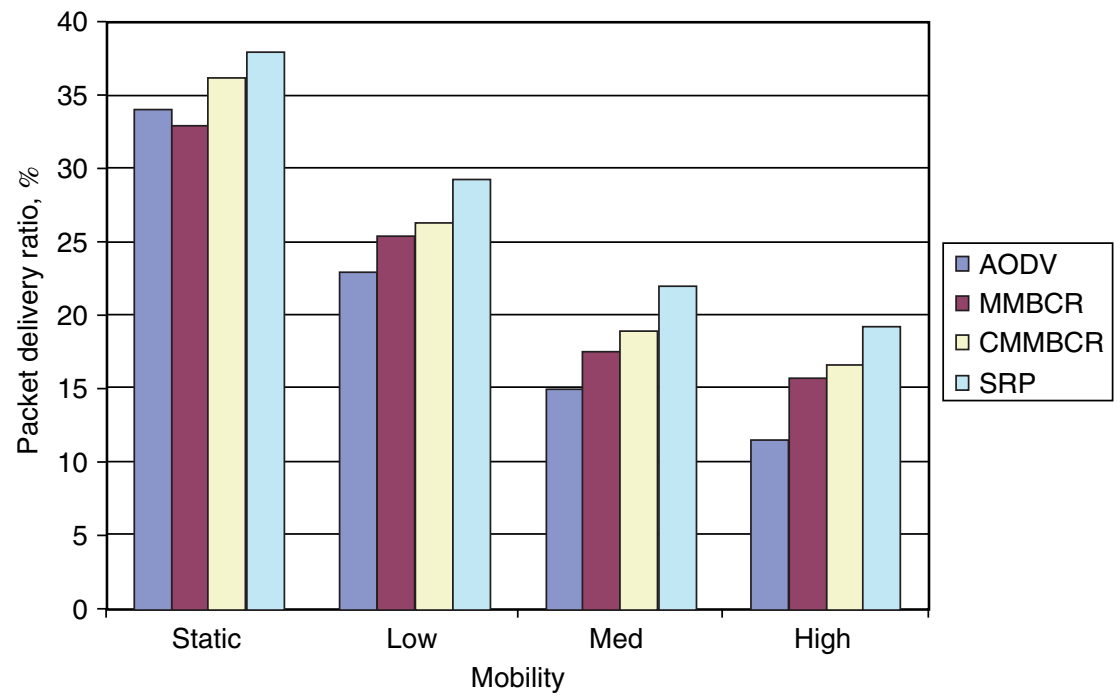

Figure 9. Packet delivery ratio at $56 \mathrm{kbps}$ traffic rate.

highercompared to the PDRs of CMMBCR, MMBCR and AODV, respectively, at static (no mobility) $56 \mathrm{kbps}$ traffic rate.

The current results show that PDRs of all four protocols decrease as the mobility and traffic density increases. However, SRP outperforms CMMBCR, MMBCR and AODV in different mobility and traffic ratewithin the 5\% error margin with reference to the Figure 8 and Figure 9. During simulation, we ran 30 simulations (with different seeds) to obtain the average value for each data point with the setting of 95 percent confidence intervals of all results.

\subsection{Average End-To-End Delay}

Figure 10 elucidates the effect of mobility on Average End-to-End Delay (AEED) of the SRP, CMMBCR, MMBCR and AODV protocols. The AEED of SRP is 79.9\% and 72.4\% smaller compared to AEED of CMMBCR and MMBCR, respectively, at a high mobility for $8 \mathrm{kbps}$ traffic rate. It is also observed that the AEED of SRP is $47.5 \%$ higher than that of AODV at a high mobility for $8 \mathrm{kbps}$ traffic rate. The AEED of SRP is $55.6 \%$ and $47.9 \%$ lower than that of CMMBCR and MMBCR, and $48.9 \%$ higher than that of AODV at a medium mobility for $8 \mathrm{kbps}$ traffic rate. The AEED of SRP is $39.3 \%$ and $25.8 \%$ lower than that of CMMBCR and MMBCR, respectively, at a low mobility for $8 \mathrm{kbps}$ traffic.

It is also shown that the AEED of SRP is $38.7 \%$ higher than that of AODV at a low mobility for $8 \mathrm{kbps}$ traffic rate. The AEED of SRP is $88.8 \%$ and $66.7 \%$ smaller than that of CMMBCR and MMBCR, respectively, at no mobility for $8 \mathrm{kbps}$ traffic rate. The AEED of SRP is $50 \%$ higher than the AEED of AODV at a static scenario for $8 \mathrm{kbps}$ traffic rate. 


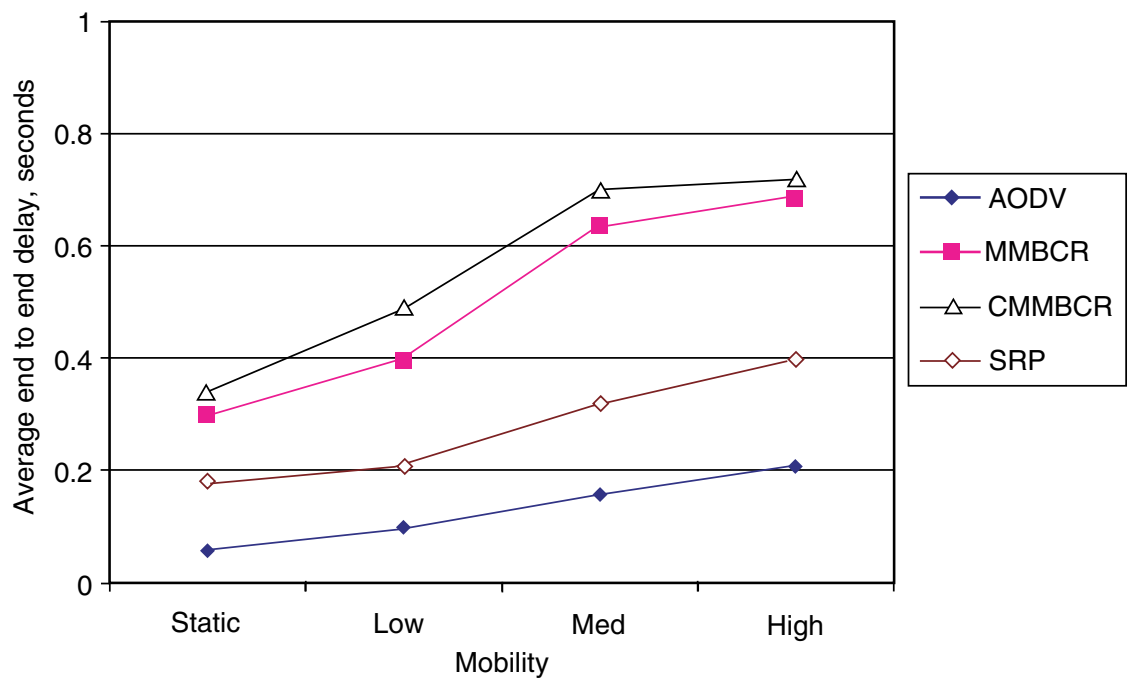

Figure 10. Average end-to-end delay at $8 \mathrm{kbps}$ traffic rate.

Figure 11 shows that the AEED of SRP is $45.7 \%$ and $38.9 \%$ smaller than that CMMBCR and MMBCR, respectively, at a high mobility for $56 \mathrm{kbps}$ traffic rate. The AEED of SRP is $32.2 \%$ higher than that of AODV at a high mobility for 56 kbps traffic rate. The AEED of SRP is $64 \%$ and $57.1 \%$ than that of CMMBCR and MMBCR, respectively, at a medium mobility for 56 kbps traffic. The AEED of SRP is $11.1 \%$ lower than that of AODV at a medium mobility for $56 \mathrm{kbps}$ traffic rate. The AEED of

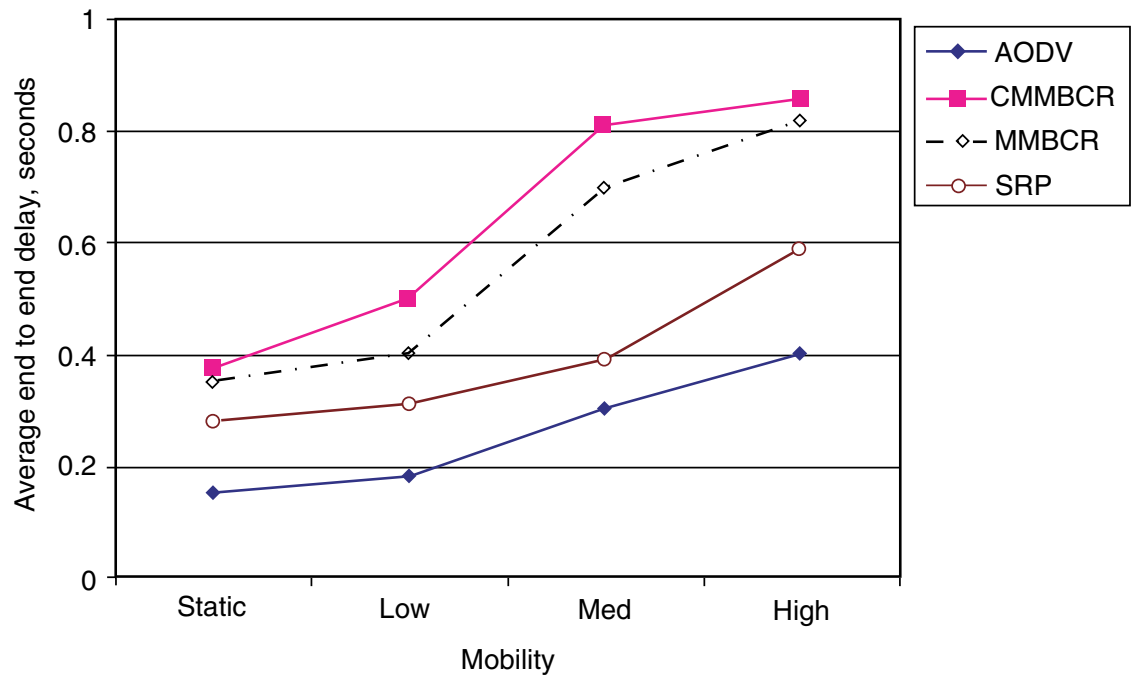

Figure 11. Average end-to-end delay at $56 \mathrm{kbps}$ trafficrate. 
SRP is $61 \%$ and $29 \%$ lower than that of CMMBCR and MMBCR, respectively, at a low mobility for $56 \mathrm{kbps}$ traffic rate. The AEED of SRP is $41 \%$ higher than that of AODV at a low mobility for $56 \mathrm{kbps}$ traffic rate. The AEED of SRP is $24.9 \%$ and $33.9 \%$ lower than that of CMMBCR and MMBCR, respectively, at no mobility for $56 \mathrm{kbps}$ traffic rate. The AEED of SRP is $46 \%$ higher than that of AODV at a static scenario for 56 kbps traffic rate.

From Figure 10 and Figure 11, it is noticed that the AEED of all the four protocols increase as the mobility and traffic rate increase. AODV has less delay than SRP, CMMBCR and MMBCR as it takes the shortest path in both low traffic and high traffic network. This is the trade-off for a smart routing protocol relative to the traditional and shortest path routing protocol such as AODV.

\subsection{Average Remaining Battery Capacity}

Figure 12 demonstrates that the percentage of average remaining battery capacity of the nodes for all protocols decreases with the simulation time for the high mobility in $8 \mathrm{kbps}$ traffic network.

The simulation result shows that SRP is better than CMMBCR, MMBCR and AODV in sustaining the remaining battery capacity above $50 \%$ for a long time. The remaining battery capacity of SRP decreases almost linearly until 3000 seconds, and decreases rapidly afterward. In comparison, the remaining battery capacity of both CMMBCR, MMBCR decreases rapidly earlier from 2500 seconds, and that of AODV decreases rapidly from 2000 seconds, respectively.

It is also observed that at 3000 seconds, SRP sustains a remaining battery capacity of $56 \%$, whereas CMMBCR maintains only $36 \%$, MMBCR maintains only $32 \%$ and AODV maintains only $29 \%$. This is because the nodes near the source nodes consume a large amount of battery power to forward data packets from a source node which is

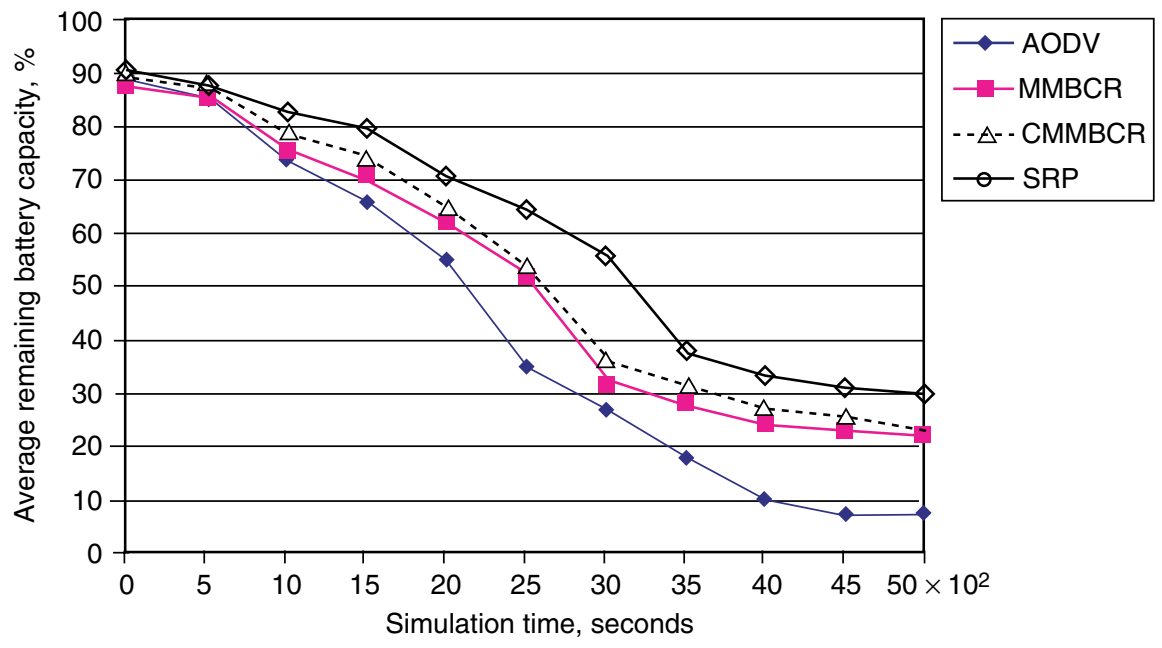

Figure 12. Remaining battery capacity for high mobility $8 \mathrm{kbps}$ traffic rate. 
located far away from the destination node. Therefore, the intermediate nodes far away from the destination node cannot find the route to the destination node quickly. If the route is not found, each node tries to find it again and again. As a result, many nodes consume a large amount of battery power to find the route to the destination nodes. On the contrary, the remaining battery capacity of the proposed SRP protocol decreases almost linearly. It is because the intermediate node with lower remaining battery capacity cancels the retransmission of the RREQ packet. Consequently, a small amount of battery power is consumed when the route delivery process is tried again. Moreover, the remaining battery capacity of the CMMBCR and MMBCR protocol also decrease gradually. Because some intermediate nodes with a higher battery capacity assist the data packets forwarded to the destination node, each node can find the route to the destination nodes easily.

Figure 13 shows the percentage of the average remaining battery capacity of the nodes decreasing with the simulation time for the high mobility $56 \mathrm{kbps}$ traffic network. Similar to Figure 12, it is seen that SRP is better than CMMBCR, MMBCR and AODV in sustaining the remaining battery capacity above $50 \%$ for a long time even in a high mobility and high traffic scenario. For instance, SRP maintains are maining battery capacity of $56 \%$, where as CMMBCR maintains only $42 \%$, MMBCR maintains only $40 \%$ and AODV maintains only $33 \%$ at 2500 seconds.

\subsection{Active Cooperative Nodes}

Figure 14 shows the percentage of active cooperative nodes with respect to simulation time for high mobility and $8 \mathrm{kbps}$ traffic network.

It is indicated that the percentage of active cooperative nodes decreases with the simulation time. Besides, SRP has more than $80 \%$ of active cooperative nodes compared to CMMBCR, MMBCR and AODV for long simulation time. At 3500

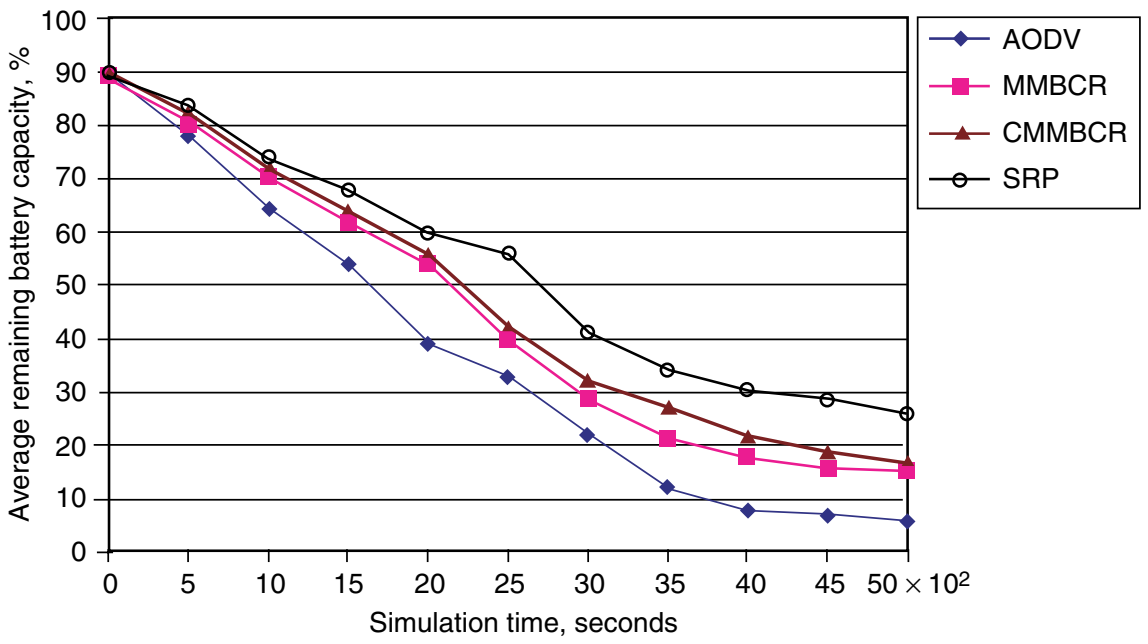

Figure 13. Remaining battery capacity for high mobility $56 \mathrm{kbps}$ traffic rate. 


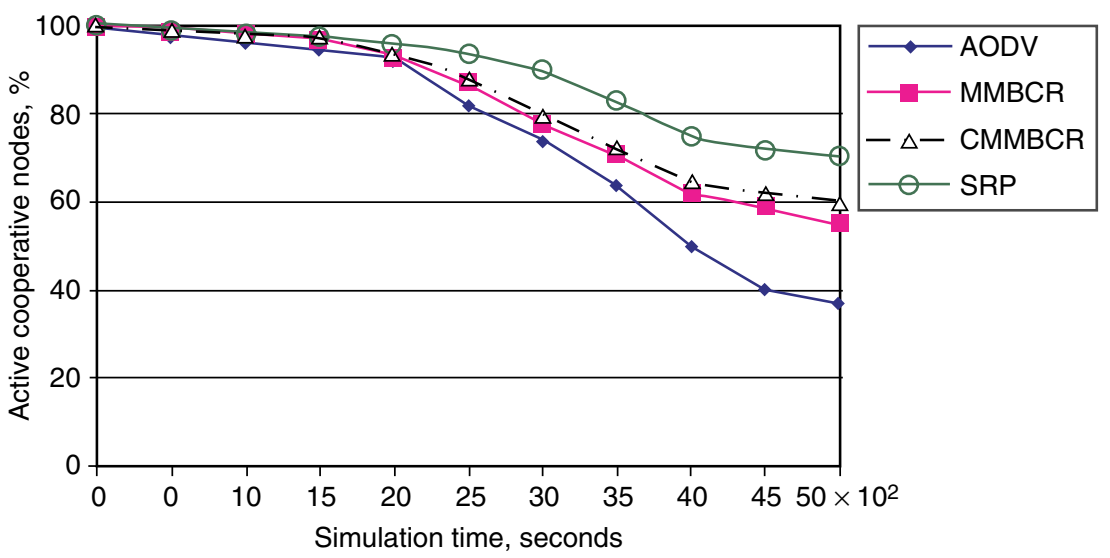

Figure 14. Active cooperative nodes for high mobility $8 \mathrm{kbps}$ traffic rate.

seconds, SRP has $83.1 \%$ of active cooperative nodes, where as CMMBCR has only $72.2 \%$, MMBCR has only $71 \%$, and AODV maintains only $64 \%$ of active cooperative nodes.

Figure 14 also shows that the percentage of active cooperative nodes of AODV decreases rapidly from 2000 seconds. This is because the nodes consume a large amount of battery power to find the route to the destination nodes. Therefore, many nodes become inactive even if the RREQs fail to reach the destination nodes. Even though the percentage of active cooperative nodes of the SRP protocol decreases rapidly beyond 3000 seconds, it still keeps $90 \%$ of the cooperative nodes as the SRP cannot find the route to the destination node from 3000 seconds. Therefore, most of the intermediate nodes do not consume much battery power. On the other hand, the percentages of active cooperative nodes of CMMBCR and MMBCR protocols decrease withthe simulation time. This is because the intermediate nodes, which assist to construct the route to the destination node and almost all the nodes, can communicate with the destination node.

Figure 15 presents the data of percentage of active cooperative nodes with respect to simulation time for high mobility and 56 kbps traffic network. It is found from Figures 14 and 15 that the percentage of active cooperative nodes decreases as the traffic rate and the simulation time increase. It is also observed that the SRP has more than $60 \%$ of active cooperative nodes compared to CMMBCR, MMBCR and AODV for a long simulation time. At 2500 seconds, SRP has the $66 \%$ of active cooperative nodes, where as CMMBCR has only 55\%, MMBCR has only 53\%, and AODV maintains only $45.4 \%$ of active cooperative nodes.

\subsubsection{Communication Reliability (CR)}

Communication Reliability $(\mathrm{CR})$ is one of the prime measures to validate the performance of wireless medical networks. However, it is critical to understand that the $\mathrm{CR}$ of wireless medical networks depends on the number of Active Cooperative Nodes 


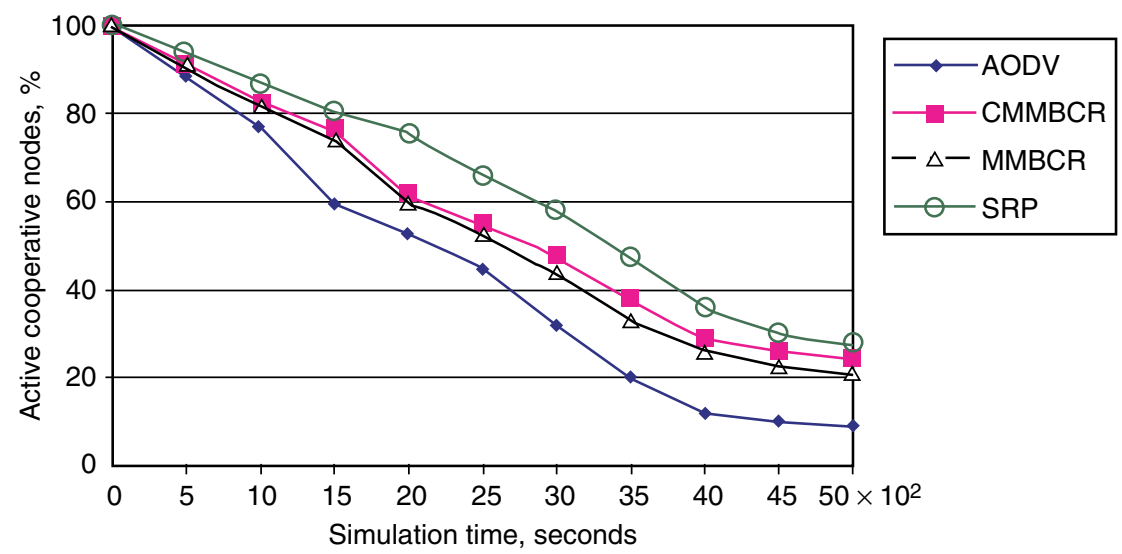

Figure 15. Active cooperative nodes for high mobility $56 \mathrm{kbps}$ traffic rate.

(ACN) in InterBAN communication networks. In this paper, we consider multi-hop wireless access networks. For a wireless link, the longer the distance between the source and the destination, the higher the probability of packet error. If a relay is introduced, the transmission range of a single hop is reduced, but the number of hops increases, which makes the hop-by-hop transmission more complex and error prone [26]. Unlike a single-hop network, in a multi-hop network, a packet may be relayed by intermediate nodes before it arrives at the destination. In multi-hop networks, the end-to-end outage probability in sending or receiving a correct packet to or from the destination node is determined by two factors: the number of hops along its packet routing path and the probability of link existence for each hop.

In the current work, $\mathrm{CR}$ is observed based on the number of active nodes at a given time instant. We assumed that the simulation time (Maximum: 0-5000 seconds), network coverage area $(800 \mathrm{~m} \times 800 \mathrm{~m})$, average path length (1.5), average degree (2.4) and probability of link existence $(0.5)$ for both $8 \mathrm{kbps}$ and $56 \mathrm{kbps}$ traffic scenarios. Table 2 shows $\%$ CR of all the four protocols at a low density and high mobility traffic

Table 2. Percentage of communication reliability (CR) at $8 \mathrm{kbps}$, high mobility traffic

\begin{tabular}{|c|c|c|c|c|c|c|c|c|}
\hline \multirow{2}{*}{$\begin{array}{l}\text { Simulation } \\
\text { Time }\left(10^{2} \mathrm{sec}\right)\end{array}$} & \multicolumn{2}{|c|}{ SRP } & \multicolumn{2}{|c|}{ CMMBCR } & \multicolumn{2}{|c|}{ MMBCR } & \multicolumn{2}{|c|}{ AODV } \\
\hline & $\mathrm{ACN}$ & CR & $\mathrm{ACN}$ & CR & $\mathrm{ACN}$ & CR & $\mathrm{ACN}$ & CR \\
\hline 0 & 100 & 1 & 100 & 1 & 100 & 1 & 100 & 1 \\
\hline 10 & 98.6 & 0.99 & 98.3 & 0.87 & 98.2 & 0.86 & 96.4 & 0.81 \\
\hline 20 & 96 & 0.92 & 94 & 0.82 & 93.1 & 0.80 & 93 & 0.76 \\
\hline 30 & 90 & 0.90 & 79.6 & 0.71 & 78 & 0.69 & 74 & 0.62 \\
\hline 40 & 74.9 & 0.72 & 64.5 & 0.58 & 62 & 0.57 & 50 & 0.52 \\
\hline 50 & 70.4 & 0.66 & 60 & 0.51 & 55 & 0.50 & 37 & 0.49 \\
\hline
\end{tabular}


Table 3. Percentage of communication reliability (CR) at $56 \mathrm{kbps}$, high mobility traffic

\begin{tabular}{|c|c|c|c|c|c|c|c|c|}
\hline \multirow{2}{*}{$\begin{array}{l}\text { Simulation } \\
\text { Time }\left(10^{2} \mathrm{sec}\right)\end{array}$} & \multicolumn{2}{|c|}{ SRP } & \multicolumn{2}{|c|}{ CMMBCR } & \multicolumn{2}{|c|}{ MMBCR } & \multicolumn{2}{|c|}{ AODV } \\
\hline & $\mathrm{ACN}$ & CR & ACN & CR & $\mathrm{ACN}$ & CR & $\mathrm{ACN}$ & CR \\
\hline 0 & 100 & 1 & 100 & 1 & 100 & 1 & 100 & 1 \\
\hline 10 & 87 & 0.94 & 98.3 & 0.83 & 98.2 & 0.82 & 98.2 & 0.79 \\
\hline 20 & 76 & 0.89 & 94 & 0.76 & 93.1 & 0.73 & 93.1 & 0.70 \\
\hline 30 & 58 & 0.82 & 79.6 & 0.69 & 78 & 0.63 & 78 & 0.61 \\
\hline 40 & 36 & 0.70 & 64.5 & 0.56 & 62 & 0.51 & 62 & 0.48 \\
\hline 50 & 28 & 0.62 & 60 & 0.50 & 55 & 0.49 & 55 & 0.41 \\
\hline
\end{tabular}

scenario. It is observed that the percentage of CR decreases with the simulation time. However, SRP maintains more than $90 \%$ of CR relative to CMMBCR, MMBCR and AODV for long simulation time. At 3000 seconds, SRP has $90 \%$ of the CR, where as CMMBCR has only $71 \%$, MMBCR has only $69 \%$, and AODV maintains only $52 \%$ of CR.

Table 3 shows percentage CR of all the four protocols at a high density and high mobility traffic scenario. It is found that the percentage of CR decreases with the simulation time. SRP maintains more than $80 \%$ of CR relative to CMMBCR, MMBCR and AODV for long simulation time. At 3000 seconds,SRP maintains $82 \%$ of the CR, whereas CMMBCR has only 69\%, MMBCR has only 63\%, and AODV maintains only $61 \%$ of CR. This is due to the difference in the number of active nodes involved in constructing reliable paths between the source and the destination among these protocols.

\subsection{Number of Route Constructions}

Figure 16 presents the number of route constructions by AODV, MMBCR and SRP routing protocols during an event. The event might be a route initiation or link failure. It is observed that AODV has to build 3.6 routes, compared to 1.9 routes for CMMBCR; 1.4 routes for MMBCR, and 1.1 routesfor SRP for each event to occur. Figure 17 depicts a similar trend for high mobility and high traffic rate, and demonstrates that AODV has to build 9.2 routes,compared to 5 routesfor CMMBCR, 4.8 routes for MMBCR, and 3.1 routes for SRP, for each event to occur.

Figures 16 and 17 showed that AODV makes more trialsthan other protocols to construct the route to the destination node for different traffic rate at high mobility. It is because AODV may use the intermediate node with a small amount of remaining battery power to construct the route. Therefore, several route reconstructions are required. On the contrary, SRP keeps a high active cooperative node rate by conserving the remaining battery power. As a result, SRP can construct the route to the destination node more effectively as shown by the smaller number of route constructions. 


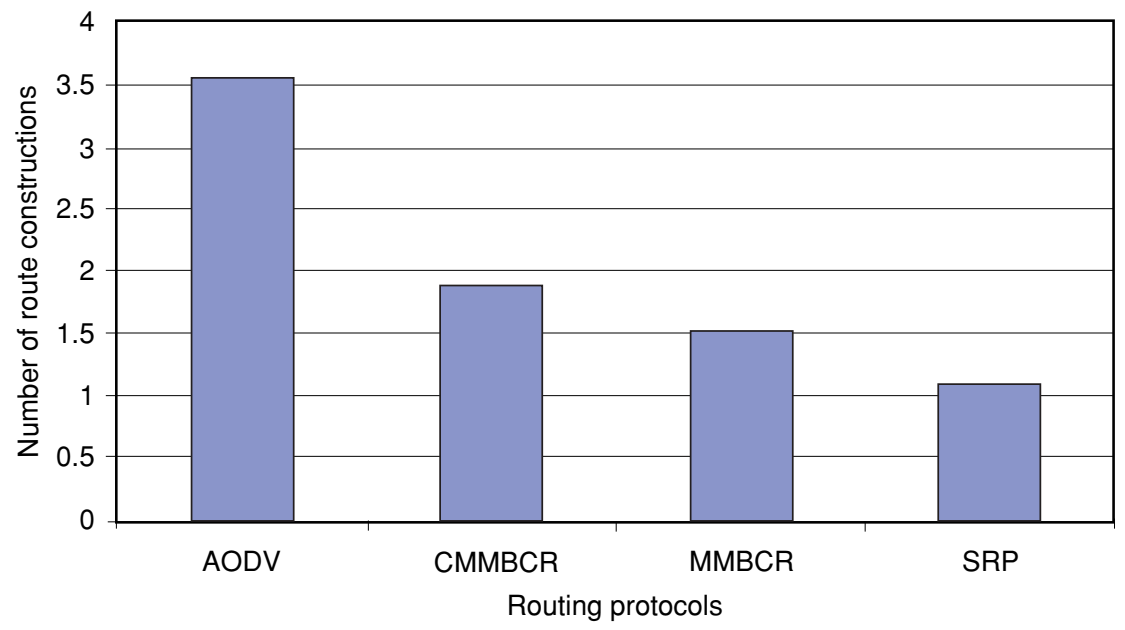

Figure 16. Number of route constructions for high mobility $8 \mathrm{kbps}$ traffic rate.

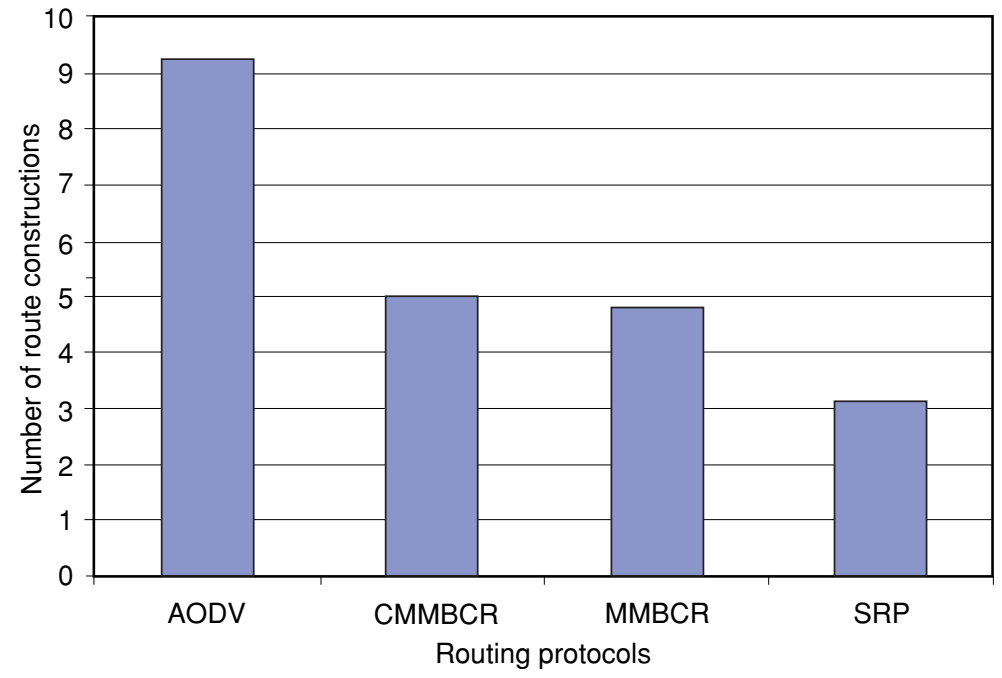

Figure 17. Number of route constructions for high mobility 56 kbps traffic rate.

\subsection{Routing Overhead}

Figure 18 demonstrates the effect of mobility on Routing Overhead (RO) of the SRP, CMMBCR, MMBCR and AODV protocols for $8 \mathrm{kbps}$ traffic rate. The RO of SRP is $9.7 \%, 14.1 \%$, and $23.4 \%$ higher than that of CMMBCR, MMBCR and AODV, respectively, at a high mobility for $8 \mathrm{kbps}$ traffic rate. The RO of SRP is $6.4 \%, 13.7 \%$, and $19.95 \%$ higher than that of CMMBCR, MMBCR and AODV, respectively, at a medium mobility for $8 \mathrm{kbps}$ traffic rate. 


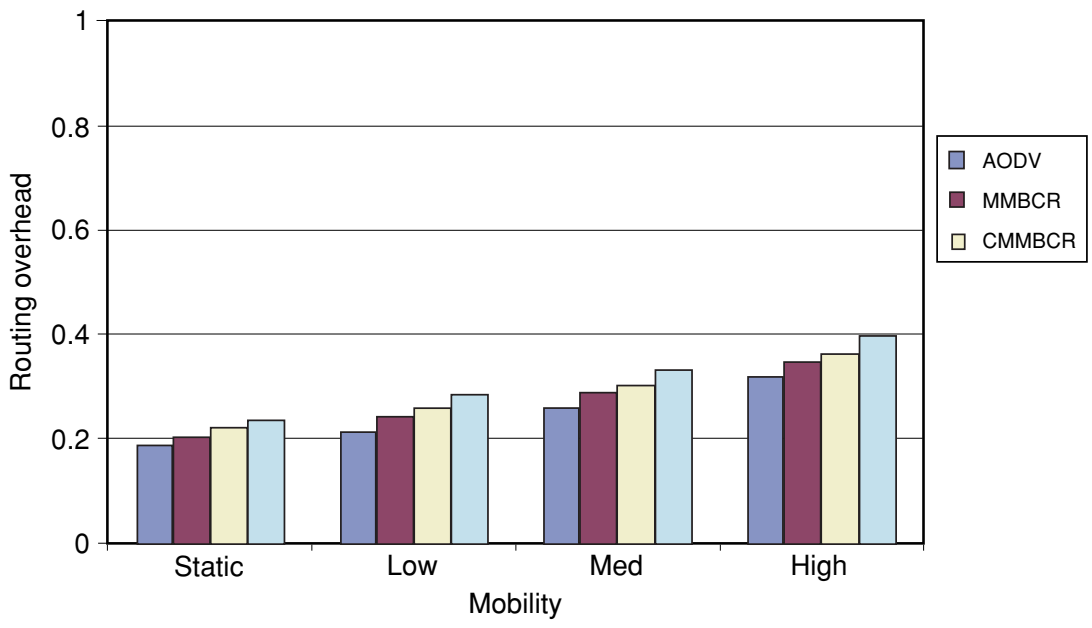

Figure 18. Routing overhead for high mobility $8 \mathrm{kbps}$ traffic rate.

The RO of SRP is $4.8 \%, 10.2 \%$, and $15.7 \%$ higher than that of CMMBCR, MMBCR, and AODV, respectively, at a low mobility in $8 \mathrm{kbps}$ traffic rate. The RO of SRP is $1.7 \%, 6.8 \%$, and $11.9 \%$ higher than that of CMMBCR, MMBCR, and AODV, respectively, at a static scenario for $8 \mathrm{kbps}$ traffic rate.

Figure 19 shows the effect of mobility on Routing Overhead (RO) of the SRP, CMMBCR, MMBCR and AODV protocols for $56 \mathrm{kbps}$ traffic rate. The RO of SRP is $12.7 \%, 19.7 \%$, and $24.3 \%$ higher than that of CMMBCR, MMBCR, and AODV, respectively, at a high mobility for $56 \mathrm{kbps}$ traffic rate. The RO of SRP is 3.7\%, 6.6\%, and $9.7 \%$ higher than that of CMMBCR, MMBCR, and AODV, respectively, at a

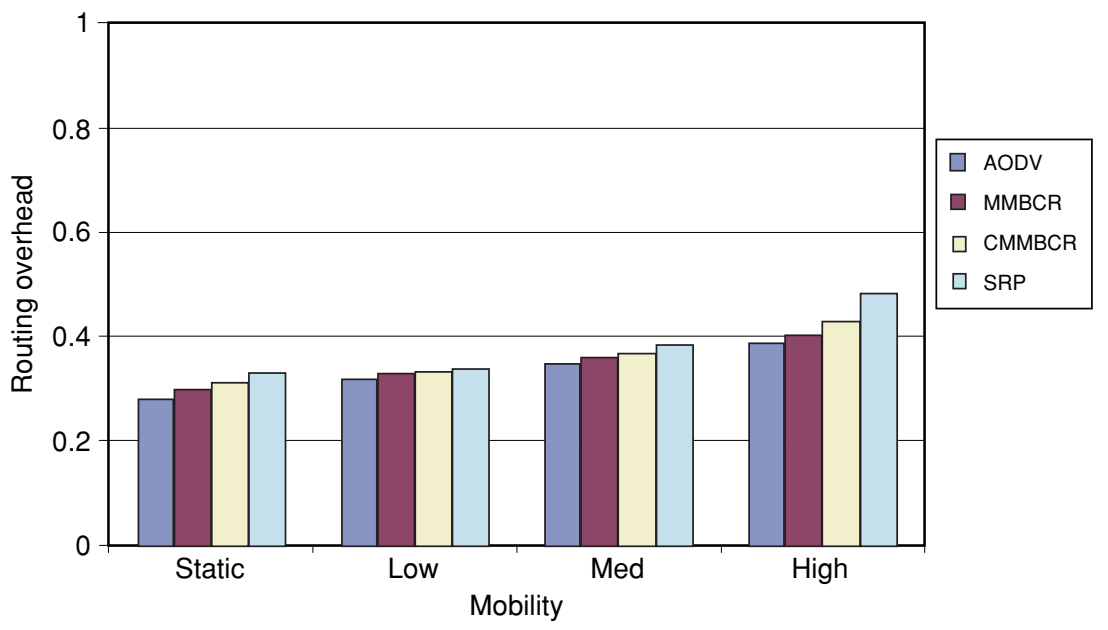

Figure 19. Routing overhead for 56 kbps traffic with different mobility levels. 
medium mobility for $56 \mathrm{kbps}$ traffic rate. The RO of SRP is $1.18 \%, 3 \%$, and $6.2 \%$ higher than thatof CMMBCR, MMBCR, and AODV, respectively, at a low mobility for $56 \mathrm{kbps}$ traffic rate. The RO of SRP is $1.2 \%, 2.4 \%$, and $6.4 \%$ higher than that CMMBCR, MMBCR and AODV respectively, at no mobility for $56 \mathrm{kbps}$ traffic rate.

Figure 18 and Figure 19 demonstrate that RO of all the four protocols increases with mobility and traffic rate. It is also observed that SRP addsrouting overhead relative to CMMBCR, MMBCR, and AODV protocols at all levels of mobility, because more rebroadcast increases the routing overhead due to more route discovery process. The added overhead does not affect the packet delivery ratio significantly as the number of reliable nodes increases in the network. However, it is noted that this performance trade-off is sensible when the network reliability is of highest concern.

\section{CONCLUSIONS}

In recent years, the electronic health (e-health) concept has evolved from telehealth to a mobile health (m-health) paradigm, enabling long-term ambulatory monitoring, and point-of-care.The proposed SRP has been developed to monitorphysiological signals from patients in the waiting areas of emergency departments. The SRP protocol canbe used to collect data from different patients' waiting in an Emergency room, and wirelessly send it to acentral computer that collects and analyzes the data. Selfishness is a new problem that arises specifically in the context of SRP MANET. The nodes belonging to multiple administrative domains may refuse to provide the necessary services for the benefit of other nodes in order to save their own resources, such as battery power. Node misbehavior that affects network operations may range from simple selfishness or lack of cooperation due to the need for power saving to active attacks such as Denial of Service (DoS). The basic idea of the proposed SRP is to keep track of power consumption in the routing of packets accordingto the recent traffic density at each node. It also exemplified how to exploit the charge recovery effect phenomenon observed in batteries. Route selection is based on a cost metric, which captures the residual battery capacity and reduces the drain rate of the mobile nodes in the network. A low-cost node is one that has a high residual battery capacity and low traffic density. Simulation results establish that network life, average remaining battery capacity, active cooperative nodes, communication reliability, number of route constructions are improved to prolong the cooperativeness of the nodes. The packet delivery ratio and network life time of the SRP are better than CMMBCR, MMBCR, and AODV. However, making routing decisions at each hop causing some additional delay, and routing overhead in routing are limitations of the SRP.

\section{CONFLICT OF INTEREST}

The author indicated no potential conflicts of interest.

\section{REFERENCES}

[1] Min Chen, Sergio Gonzalez, Athanasios Vasilakos, Huasong Cao, Victor C. M. Leung. Body Area Networks: A Survey.Mobile Network Application. 2011, 171-193.

[2] Gao T, Massey T, Selavo L, Crawford D, Chen B, Lorincz K, Shnayder V, Hauenstein L, Dabiri F, Jeng J, Chanmugam A, White D, Sarrafzadeh M, Welsh M. The advanced health and disaster aid network: a light-weight wireless medical system for triage. IEEE Trans Biomed Circuits Syst. 2007, 203-216. 
[3] Mehdi Kargar and Mohammad Ghodsi.Truthful and Secure Routing in Ad Hoc Networks with Malicious and Selfish Nodes.International Journal of Security and its Applications, 2009, 117-128.

[4] Rappaport, T. S. Wireless Communications: Principles and Practice, Prentice-Hall, Englewood Clifis, NJ, 2002.

[5] Michiardi, P. and Molva, R. Core: A Collaborative Reputation Mechanism to Enforce Node Cooperation in Mobile Ad-Hoc Network., Proc. 6th IFIP Communication and Multimedia Security Conference. 2002, 107-121.

[6] Kim, J. and Bentley, P. An Evaluation of Negative Selection in an Artificial Immune System for Network Intrusion Detection.Proc. Genetic and Evolutionary Computation Conference, Morgan Kaufmann. 2001, 1330-1337.

[7] Toh, C. K. Maximum Battery Life Routing to Support Ubiquitous Mobile Computing in Wireless Ad Hoc Networks,.IEEE Communications Magazine. 2001, 138-147.

[8] L Xiang, J Luo, C Rosenberg, Compressed Data Aggregation for Energy Efficient Wireless Sensor Networks, IEEE SECON, 2011, 46-54.

[9] Yun-Sheng Yen, Han-Chieh Chao, Ruay-Shiung Chang, Athanasios Vasilakos, Flooding-Limited and Multi-Constrained QOS Multicast Routing based on the Genetic Algorithm for MANETs, Mathematical and Computer Modelling, 2011, 2238-2250.

[10] Lijuan Cao Sharif, K. and Yu Wang Dahlberg, T. Adaptive Multiple Metrics Routing Protocols for Heterogeneous Multi-Hop Wireless Networks.Proc. Consumer Communications and Networking Conference. 2008, 13-17.

[11] N. Chilamkurti, S. Zeadally, A. Vasilakos, and V. Sharma, Cross-Layer Support for Energy Efficient Routing in Wireless Sensor Networks, Journal of Sensors, 2009, 1-9.

[12] Srinivasan, V., Nuggehalli, P., Chiasserini, C. F. and Rao, R. R. Energy Efficiency of Ad Hoc Wireless Networks with Selfish Users.Proc. European Wireless conference, 2002, 71-78.

[13] He, Daojing., Chen, C., Chan, S., Bu, J., \& Vasilakos, A. V., ReTrust: Attack-Resistant and Lightweight Trust Management for Medical Sensor Networks, Information Technology in Biomedicine, IEEE Transactions, 2012, 623-632.

[14] He, Daojing, Chen, C., Chan, S., Bu, J., \& Vasilakos, A. V., A Distributed Trust Evaluation Model and Its Application Scenarios for Medical Sensor Networks, Information Technology in Biomedicine, IEEE Transactions on, 2012, 1164-1175.

[15] Moustafa Youssef, Mohamed Ibrahim, Mohamed Abdelatif, Lin Chen, and Athanasios V. Vasilakos, Routing Metrics of Cognitive Radio Networks: A Survey, To appear at IEEE Communications Surveys and Tutorials, 2013.

[16] Wang, X., Vasilakos, A. V., Chen, M., Liu, Y., \& Kwon, T. T., A Survey of Green Mobile Networks: Opportunities and Challenges, Mobile Networks and Applications, 2012, 4-20.

[17] Guo, W., Xiong, N., Vasilakos, A. V., Chen, G., \& Yu, C., Distributed K-Connected Fault-Tolerant Topology Control Algorithms with PSO in Future Autonomic Sensor Systems. International Journal of Sensor Networks, 2012, 53-62.

[18] Cruz, R. L. and Santhanam, A. V. Optimal Routing, Link Scheduling, and Power Control in Multi-Hop Wireless Networks.Proc. 22nd Annual Joint Conference of the IEEE Computer and Communications Societies., 2003, 702-711.

[19] Katsuhiro Naito, Kazuo Mori and Hideo Kobayashi. Evaluation of Power Aware Routing for Sensor Networks with Forwarder Nodes.Journal of Systemics, Cybernetics and Informatics, 2008, 87-92.

[20] Saoucene Mahfoudh INRIA, Rocquencourt, Le Chesnay Cedex, France, "Energy-aware routing in wireless ad hoc and sensor networks. Proceedings of the 6th International Wireless Communications and Mobile Computing Conference.2010, 1126-1130.

[21] Hongbing Cheng, Chunming Rong, and Geng Yang. Design and Analysis of a Secure Routing Protocol Algorithm for Wireless Sensor Networks.Proceedings of the 2011 IEEE International Conference on Advanced Information Networking and Applications. 2011, 475-480. 
[22] Lahiri, K., Ragunathan A, Dey S and Panigrahi D. Battery Driven System Design: A New Frontier in Low Power Design. Proc. ASP-DAC International Conference of VLSI design. 2002, 261-267.

[23] Datta, S. and Eksiri, B. Sensor Network Routing Algorithms for Realistic Battery Model.Proceedings of the Information Fusion and Dissemination in Wireless Sensor Networks Workshop, 2005, 8-14.

[24] Afzal, M. I., Waqar Mahmood, Sheikh Muhammad Sajid and Shin Seoyong. Optical Wireless Communication and Recharging Mechanism of Wireless Sensor Network by Using CCRs.International Journal of Advanced Science and Technology, 2009, 49-60.

[25] Mall, R. and Patnaik, P.K, A novel power aware routing technique for mobile ad hoc networks.Proc. TENCON Conference. 2007,1-3.

[26] Lei Zheng, Ning Lu, Lin Cai, Reliable Wireless Communication Networks for Demand Response Control, IEEE Transaction On Smart Grid, 2013, 4(1); 133-140. 



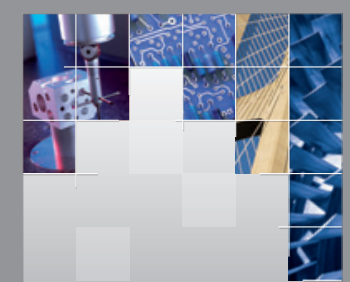

\section{Enfincering}
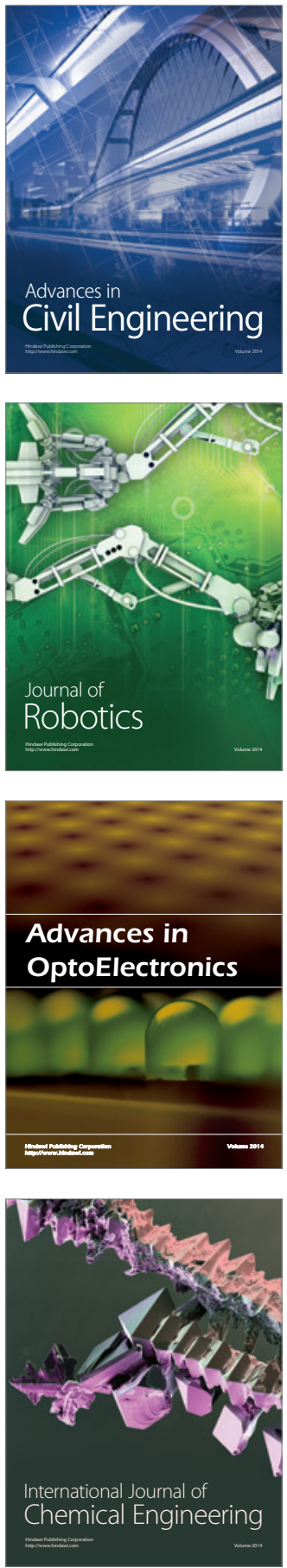

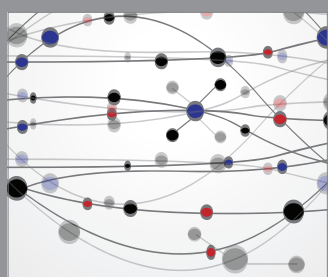

The Scientific World Journal

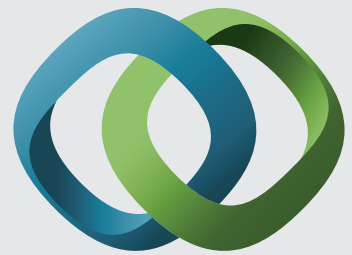

\section{Hindawi}

Submit your manuscripts at

http://www.hindawi.com
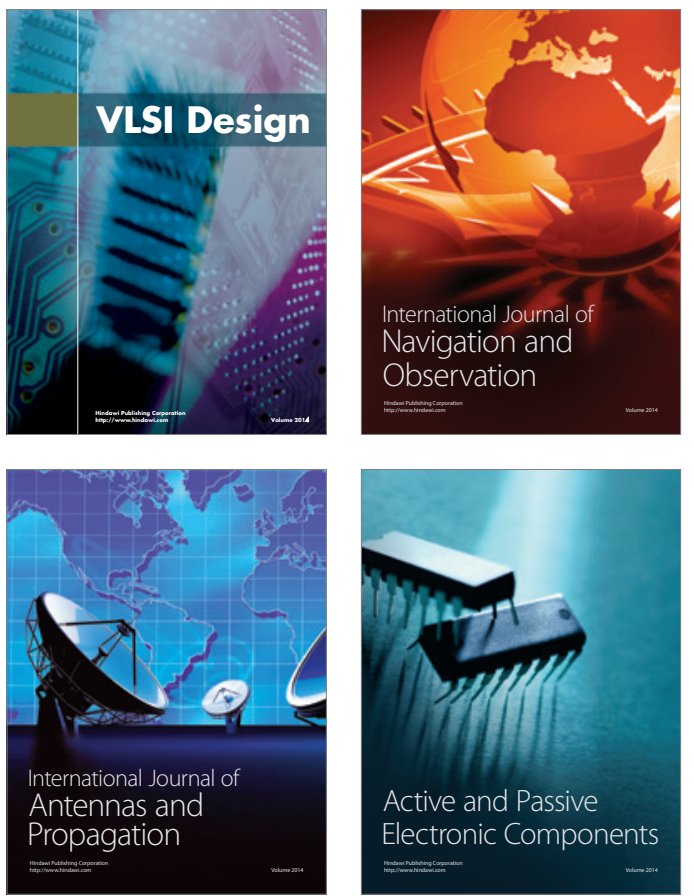
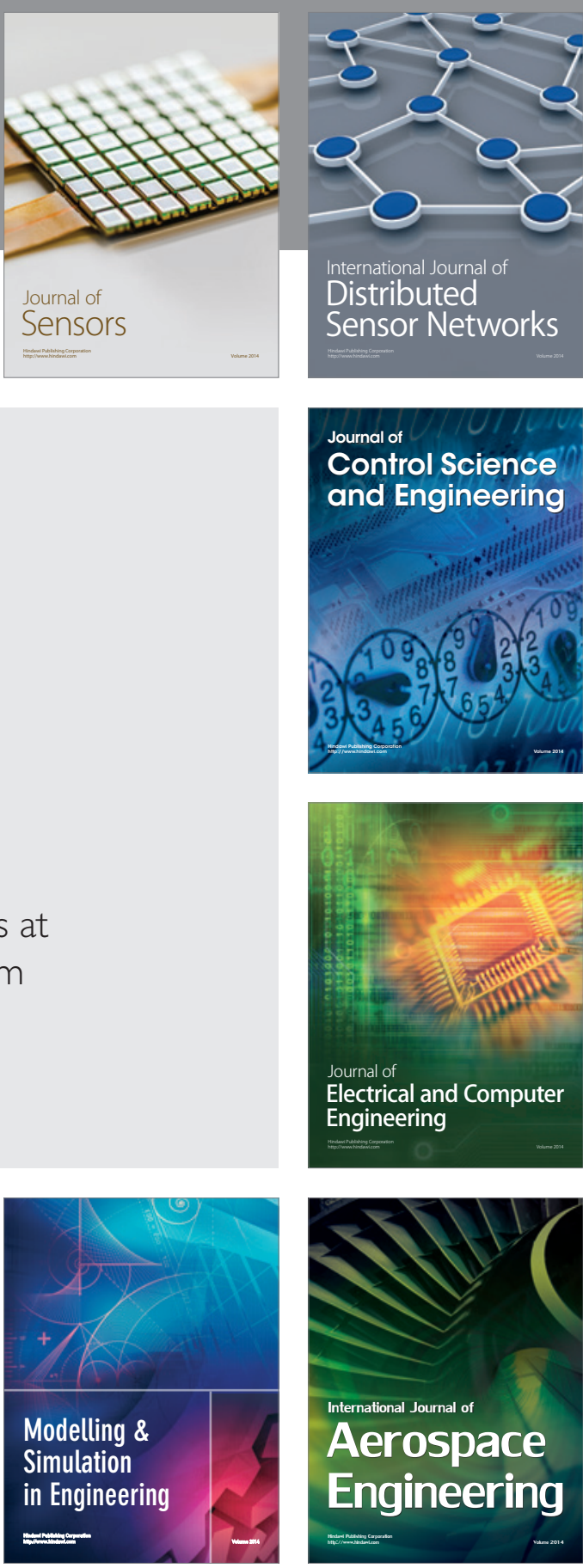

International Journal of

Distributed

Sensor Networks

Journal of

Control Science

and Engineering
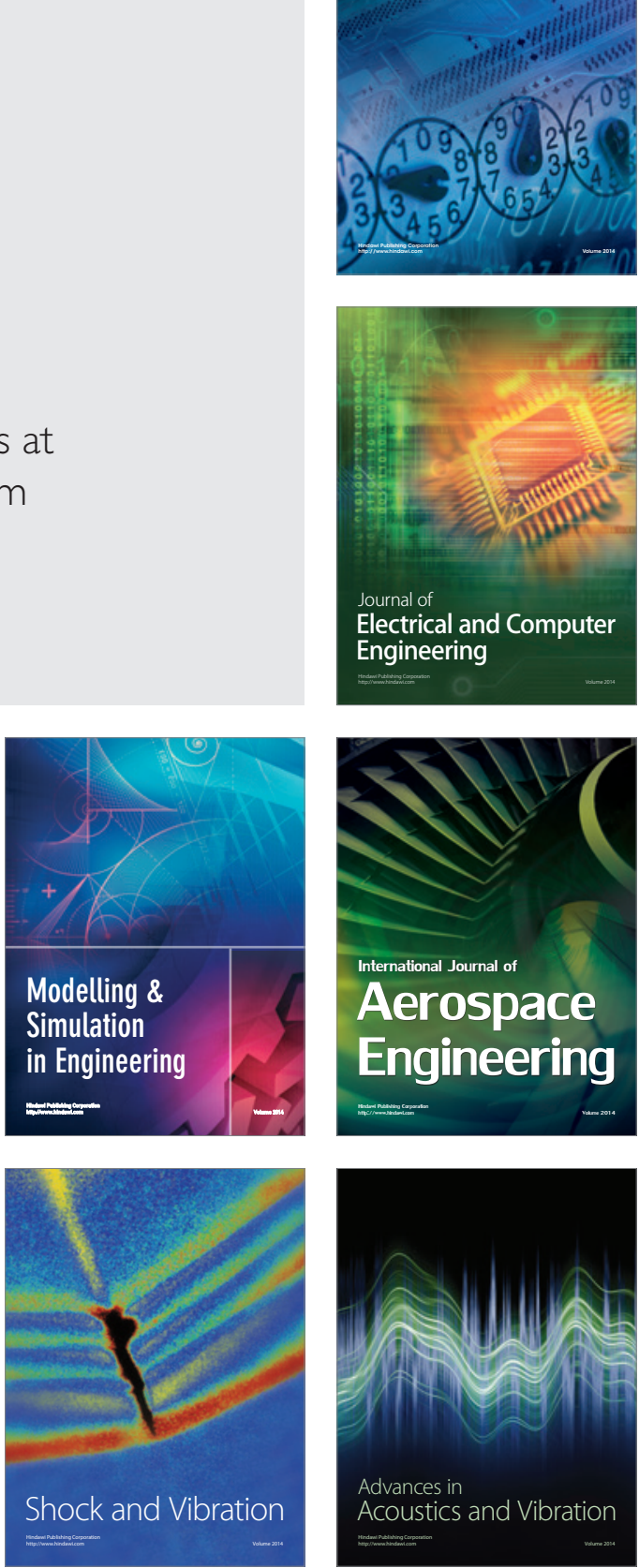